Article

\title{
New Imatinib Derivatives with Antiproliferative Activity against A549 and K562 Cancer Cells
}

\author{
Andressa Oliveira ${ }^{1,2}$, Stefany Moura ${ }^{1,2}$, Luiz Pimentel ${ }^{1}{ }^{\mathbb{C}}$, João Neto ${ }^{3}$, Rafael Dantas ${ }^{3}$, Floriano Silva-Jr ${ }^{2,3}{ }^{(\mathbb{C})}$, \\ Monica Bastos 1,2 and Nubia Boechat $1,2, *$ (i)
}

check for

updates

Citation: Oliveira, A.; Moura, S.;

Pimentel, L.; Neto, J.; Dantas, R.; Silva-Jr, F.; Bastos, M.; Boechat, N. New Imatinib Derivatives with Antiproliferative Activity against A549 and K562 Cancer Cells. Molecules 2022, 27, 750. https:// doi.org/10.3390/molecules27030750

Academic Editor: Patricia

Dias Fernandes

Received: 29 December 2021

Accepted: 13 January 2022

Published: 24 January 2022

Publisher's Note: MDPI stays neutral with regard to jurisdictional claims in published maps and institutional affiliations.

Copyright: (C) 2022 by the authors. Licensee MDPI, Basel, Switzerland. This article is an open access article distributed under the terms and conditions of the Creative Commons Attribution (CC BY) license (https:// creativecommons.org/licenses/by/ $4.0 /)$.

1 Laboratório de Sintese de Farmacos, Instituto de Tecnologia em Farmacos-Farmanguinhos, Rua Sizenando Nabuco 100, Manguinhos, Rio de Janeiro 21041-250, RJ, Brazil; andressa.oliveira@far.fiocruz.br (A.O.); stefanybazan6@far.fiocruz.br (S.M.); luiz.pimentel@far.fiocruz.br (L.P.); monica.bastos@far.fiocruz.br (M.B.)

2 Programa de Pós-graduação em Farmacologia e Química Medicinal do Instituto de Ciências Biomédicas, Centro de Ciências da Saúde, Bloco J, Ilha do Fundão, Rio de Janeiro 21941-902, RJ, Brazil; floriano@ioc.fiocruz.br

3 Laboratório de Bioquímica Experimental e Computacional de Fármacos, Instituto Oswaldo Cruz, FIOCRUZ, Av. Brasil 4365, Manguinhos, Rio de Janeiro 21040-360, RJ, Brazil; joao.neto@ioc.fiocruz.br (J.N.); rafael.dantas@ioc.fiocruz.br (R.D.)

* Correspondence: nubia.boechat@far.fiocruz.br or nboechat@gmail.com; Tel.: +55-21-3977-2465

\begin{abstract}
Tyrosine kinase enzymes are among the primary molecular targets for the treatment of some human neoplasms, such as those in lung cancer and chronic myeloid leukemia. Mutations in the enzyme domain can cause resistance and new inhibitors capable of circumventing these mutations are highly desired. The objective of this work was to synthesize and evaluate the antiproliferative ability of ten new analogs that contain isatins and the phenylamino-pyrimidine pyridine (PAPP) skeleton, the main pharmacophore group of imatinib. The 1,2,3-triazole core was used as a spacer in the derivatives through a click chemistry reaction and gave good yields. All the analogs were tested against A549 and K562 cells, lung cancer and chronic myeloid leukemia (CML) cell lines, respectively. In A549 cells, the 3,3-difluorinated compound (3a), the 5-chloro-3,3-difluorinated compound (3c) and the 5-bromo-3,3-difluorinated compound (3d) showed $\mathrm{IC}_{50}$ values of 7.2, 6.4, and 7.3 $\mu \mathrm{M}$, respectively, and were all more potent than imatinib ( $\mathrm{IC}_{50}$ of $65.4 \mu \mathrm{M}$ ). In $\mathrm{K} 562$ cells, the 3,3-difluoro-5-methylated compound ( $3 \mathbf{b}$ ) decreased cell viability to $57.5 \%$ and, at $10 \mu \mathrm{M}$, showed an $\mathrm{IC}_{50}$ value of $35.8 \mu \mathrm{M}$ (imatinib, $\mathrm{IC}_{50}=0.08 \mu \mathrm{M}$ ). The results suggest that $\mathbf{3 a}, \mathbf{3} \mathbf{c}$, and $\mathbf{3 d}$ can be used as prototypes for the development of more potent and selective derivatives against lung cancer.
\end{abstract}

Keywords: tyrosine kinase inhibitors; imatinib; K562; A549; PAPP and isatin

\section{Introduction}

Protein tyrosine kinases (PTKs) are a group of approximately 90 enzymes that play essential roles in cells, such as in the regulation of cell division, cell differentiation and morphogenesis [1]. PTKs can be divided into receptor tyrosine kinases (RTKs) and nonreceptor tyrosine kinases (NRTKs). Examples of RTKs and NRTKs are insulin receptors and growth factor receptors (GFRs), such as epidermal growth factor receptor (EGFR) and ABL1, respectively [2]. The deregulated action of these PTKs is directly related to the development of some types of cancer [3].

PTK BCR-ABL1 is not expressed in healthy organisms because it is a product of cellular deregulation, and this PTK has been described as an oncogene that is present in $95 \%$ of patients with chronic myeloid leukemia (CML) [4]. The discovery of the relationship between this TK and CML made the development of imatinib (1) possible, as the first drug to be used against the PTK BCR-ABL1, which revolutionized the treatment of CML [5]. This drug acts by competitively inhibiting ATP binding to the BCR-ABL1 enzyme, preventing 
substrate phosphorylation, blocking its activity, and avoiding transduction of the signals essential for cellular functions. However, the emergence of cases of resistance to this drug has shown the need to develop new second- and third-generation inhibitors. However, even these new tyrosine kinase inhibitors (TKIs) have shown many cases of resistance, highlighting the need for a continuous search for new compounds that can treat these resistant tumors [6-8].

EGFR is a PTK receptor, also known as ErbB1/HER1, that belongs to the ErbB family, which also includes ErbB2/HER2/Neu, ErbB3/HER3 and ErbB4/HER42. EGFR participates in cell proliferation and apoptosis and has been classified as a proto-oncogene because it is commonly seen in cancers, such as non-small-cell lung cancer, metastatic colorectal cancer, glioblastoma, head and neck cancer, pancreatic cancer and breast cancer [9]. EGFR is a key mediator that plays a crucial role in cell growth, proliferation, survival and migration. This protein belongs to the kinase family and, in recent years, the discovery of the relationship between the overexpression of EGFR and solid tumors has made EGFR a target in modern medicinal chemistry for the planning of new anticancer agents [10].

The demand imposed by frequent mutations occurring in the kinase domain is still among the greatest limitations to treatment by target therapy, especially in oncology [11]. Therefore, it is imperative to develop new TKIs that can reduce both known resistances and others that may arise.

By continuing the work that the group has been developing to obtain new imatinib analogs [12], in this work, we synthesized a new series of ten imatinib analogs (2a-e and 3a-e, Figure 1) and evaluated their cytotoxic activity in two human cancer cell lines: K562 (CML) and A549 (lung cancer). The compounds were designed as molecular hybrids containing phenylamino-pyrimidine pyridine (PAPP, in blue) and isatin (in red) scaffold units, connected by a $1 H-1,2,3$-triazole ring (in purple) (Figure 1). PAPP is an important pharmacophoric fragment of imatinib $[13,14]$. Isatins and some derivatives have relevance as TKIs [15], as shown for the cytotoxic activity of compound 4 against K562 cells, a cell lineage that has the constitutive activity of the TK BCR-ABL1. Compound 4 showed an $\mathrm{IC}_{50}$ of $0.03 \mu \mathrm{M}$, which was comparable to that obtained with the standard drug, irinotecan $\left(\mathrm{CPT}-11, \mathrm{IC}_{50}=0.07 \mu \mathrm{M}\right)$ (Figure 1) [16]. Moreover, isatin scaffolds are present in sunitinib (5) and nintedanib (6), which are important TKIs. In derivatives 2a-e and 3a-e, the isatins have different substituents, which were chosen to verify their electronic contributions as electron attractor $(\mathrm{Cl}, \mathrm{Br}, \mathrm{F})$ and electron donor $\left(\mathrm{CH}_{3}\right)$ groups. The $1 \mathrm{H}-1,2,3$-triazole ring was used as a spacer between the two units, PAPP and isatin, since this nucleus has been described in compounds with potential TK inhibitory activity, as in compound 7 [17].

The cell lines used herein (K562 and A549) are sensitive to imatinib. Although there is a vast literature showing the cytotoxicity of imatinib analogs in K562 cells, little is known about their effects in the A549 cell line. Shijie and coworkers showed that imatinib and its derivatives presented good inhibitory results in A549 cells. An example is compound 8 , which has a PAPP skeleton and decreased cell viability to $38.5 \%$ at a concentration of $150 \mu \mathrm{M}$ (Figure 1) [18]. This result encouraged us to also test our compounds against this cell line. 


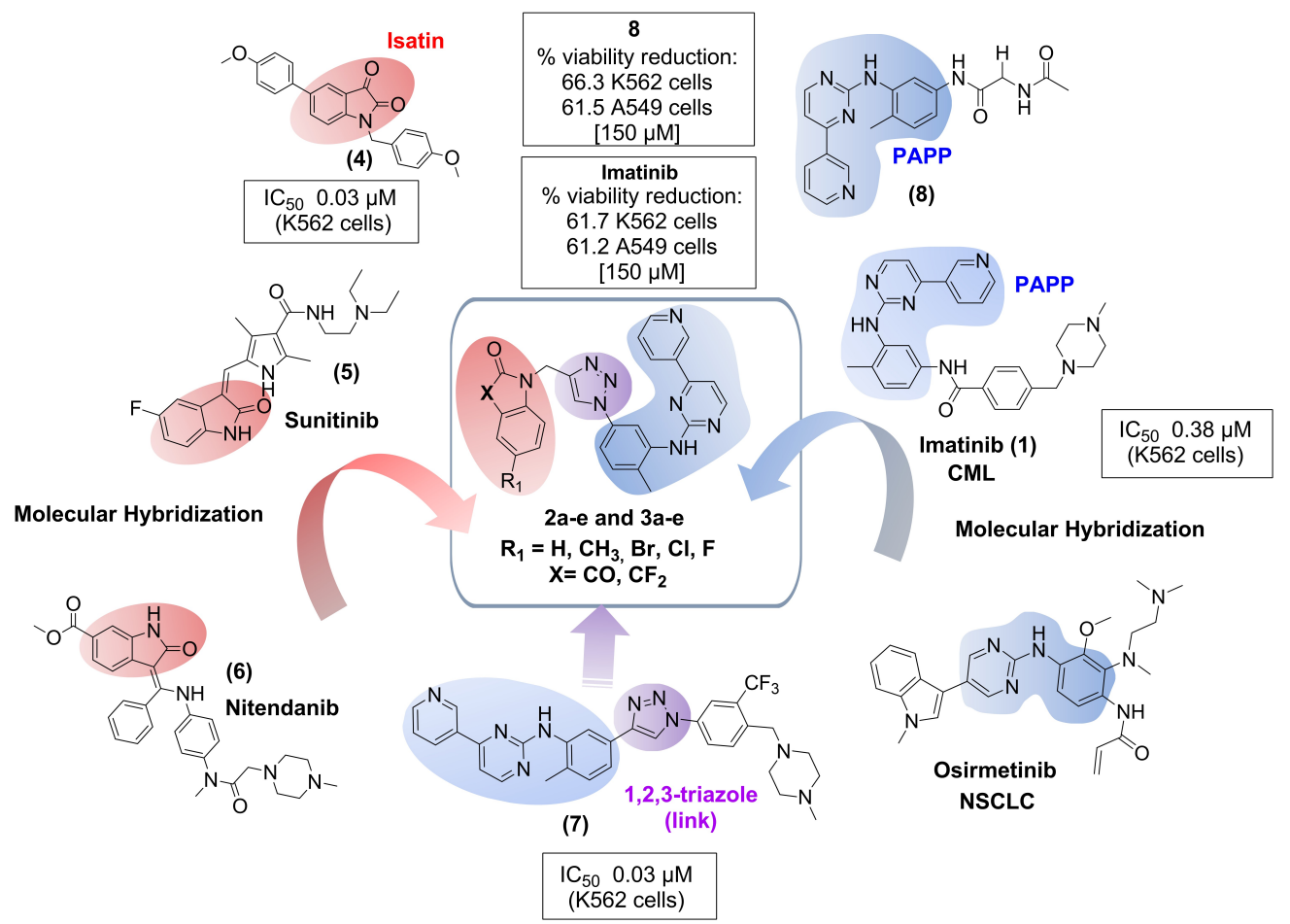

Figure 1. Design of new products $2 \mathbf{a}-\mathbf{e}$ and $3 \mathbf{a}-\mathbf{e}$.

\section{Results and Discussion}

\subsection{Chemistry}

Ten new hybrids presenting the PAPP group and isatin or derivatives spaced with a $1 H-1,2,3$-triazole ring were synthesized, as shown in Scheme 1.
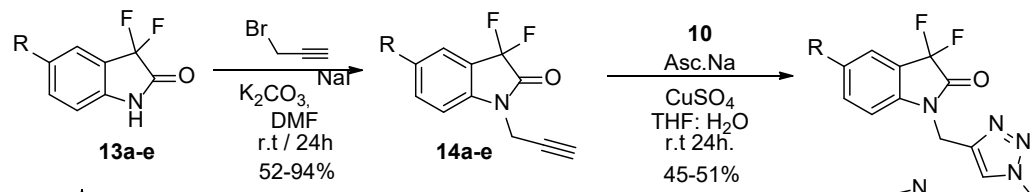

DAST
$\begin{aligned} & \mathrm{CH}_{2} \mathrm{Cl}_{2,} \text {,.t., } 4 \mathrm{~h} \\
& 68-88 \%\end{aligned}$
\begin{tabular}{|l} 
(a) $\mathrm{R}=\mathrm{H}$ \\
(b) $\mathrm{R}=\mathrm{CH}_{3}$ \\
(c) $\mathrm{R}=\mathrm{Cl}$ \\
(d) $\mathrm{R}=\mathrm{Br}$ \\
(e) $\mathrm{R}=\mathrm{F}$ \\
\hline
\end{tabular}

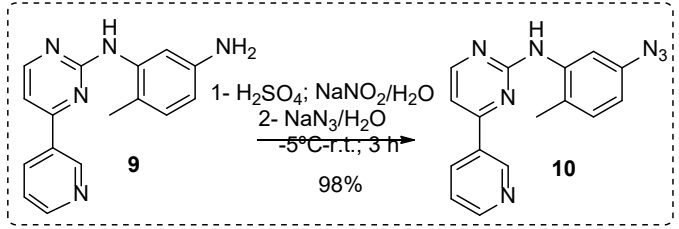<smiles>[R]c1ccc2c(c1)C(=O)C(=O)N2</smiles>
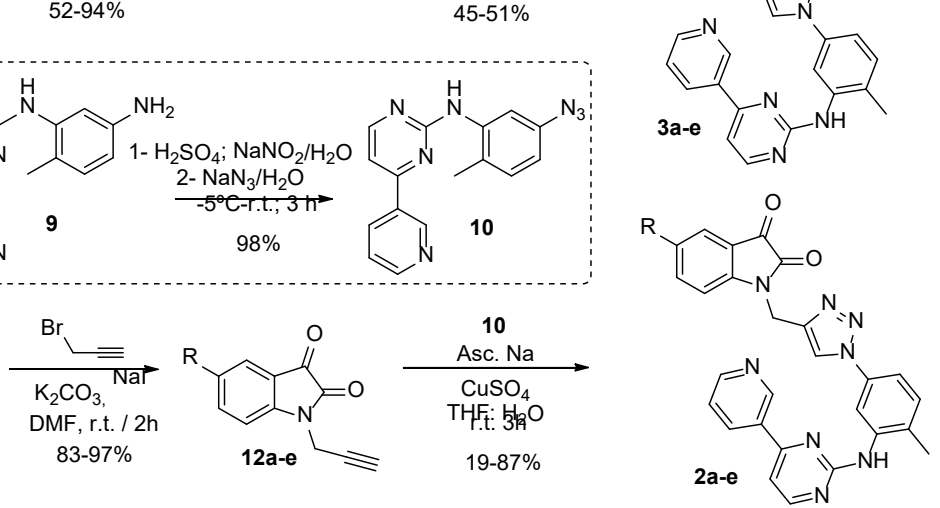

Scheme 1. Synthetic route to obtaining the final products, 2a-e and 3a-e.

Initially, commercially available isatins (11a-e) were $N$-alkylated with propargyl bromide to generate 1-propyl-indoline-2,3-dione derivatives (12a-e) at an 83-97\% yield (Scheme 1). Compounds 12a-e were characterized, and the data were in agreement with the literature [19,20]. N-(5-Azido-2-methylphenyl)-4-(pyridin-3-yl)pyrimidin-2-amine (10) was prepared via an aromatic nucleophilic substitution reaction, through the formation of a diazonium salt using intermediate 9 . This compound was obtained at a $98 \%$ yield and was characterized; the data were in agreement with the literature [19]. 
The fluorination of compounds 11a-e with diethylaminosulfurtrifluoride (DAST) in $\mathrm{CH}_{2} \mathrm{Cl}_{2}$ at $25{ }^{\circ} \mathrm{C}$ produced intermediates $13 a-e$ after $4 \mathrm{~h}$, with a $68-88 \%$ yield. The IR spectra of intermediates 13a-e showed absorption bands in the region from 3200 to $3271 \mathrm{~cm}^{-1}$, corresponding to the axial strain of the NH bond, and stretches between 1268 and $1335 \mathrm{~cm}^{-1}$, corresponding to the axial strain of the $\mathrm{CF}_{2}$ bond [19]. Spectroscopic data of intermediates 13a-d have already been described in the literature, but that of the intermediate 13e is unpublished. The ${ }^{1} \mathrm{H}$ NMR spectrum of intermediate 13e showed a singlet at $11.20 \mathrm{ppm}$ corresponding to a $\mathrm{N}-\mathrm{H}$ hydrogen. In the ${ }^{13} \mathrm{C} \mathrm{NMR}$ spectrum, a triplet at 165.7 ppm with $J=29 \mathrm{~Hz}$, corresponding to a carbonyl carbon and a triplet at 110.8 ppm with $J=247.9 \mathrm{~Hz}$, corresponding to the C-3 carbon were observed, and this multiplicity is due to carbon-fluorine coupling with the difluoromethylene group. The ${ }^{19} \mathrm{~F}$ NMR spectrum showed a chemical shift at $-111.1 \mathrm{ppm}$, corresponding to $\mathrm{CF}_{2}$, and the peak at 119.0 ppm corresponded to the C-F connection.

Gem-difluorinated intermediates 14a-e were prepared at a 52-94\% yield by the $N$-alkylation of compounds 13a-e. The IR spectra of intermediates 14a-e showed absorption bands in the region from 1276 to $1335 \mathrm{~cm}^{-1}$, corresponding to the axial deformation of $\mathrm{CF}_{2}$. For compounds $\mathbf{1 4 d - e , ~ N M R ~ a n a l y s e s ~ w e r e ~ p e r f o r m e d , ~ a s ~ t h e y ~ h a v e ~ n o t ~ y e t ~ b e e n ~}$ described in the literature. In the ${ }^{1} \mathrm{H}$ NMR analysis of intermediates $\mathbf{1 4 d}$ and $\mathbf{1 4 e}$, it is possible to observe a signal at 7.30 or $7.37 \mathrm{ppm}$, related to H-7, and a doublet at 4.60 or $4.61 \mathrm{ppm}$, with a coupling constant of $2.5 \mathrm{~Hz}$ for $\mathrm{H}-1^{\prime}$. In the ${ }^{13} \mathrm{C}$ NMR analyses of the intermediates 14d-e, signals corresponding to C-1', C-2' and C-3' were observed at $29.5 \mathrm{ppm}, 76.5 \mathrm{ppm}$, and between 75.5 and $75.6 \mathrm{ppm}$, respectively. The ${ }^{19} \mathrm{~F}$ NMR spectrum showed chemical shifts between -110.6 and -110.7 ppm corresponding to $\mathrm{CF}_{2}$.

The 1,3-dipolar cycloaddition reaction between the azide derivative (10) and the terminal alkynes 12a-e and 14a-e, was performed via click chemistry conditions, using sodium ascorbate and copper sulfate in THF $/ \mathrm{H}_{2} \mathrm{O}(1: 1)$ at room temperature for $3 \mathrm{~h}$, to obtain only one 1,4-regioisomer of the final products $\mathbf{2 a - e}$ and $3 \mathbf{a}-\mathbf{e}$, respectively.

New hybrids 2a-e and 3a-e were obtained in good yields, although some unsatisfactory yields may be associated with the low solubility of the final compounds, making their purification process difficult. The IR spectra for compounds $\mathbf{2 a - e}$ showed absorption bands between $3383 \mathrm{~cm}^{-1}$ and $3440 \mathrm{~cm}^{-1}$, corresponding to the axial deformation of the $\mathrm{N}-\mathrm{H}$ bond; 1736 and $1739 \mathrm{~cm}^{-1}$, corresponding to the axial deformation of the ketone carbonyl; and 1579 and $1618 \mathrm{~cm}^{-1}$, corresponding to the deformation of an amylic carbonyl. Compounds 3a-e showed absorption bands between 1284 and $1298 \mathrm{~cm}^{-1}$, corresponding to the axial deformation of the $\mathrm{CF}_{2}$ bond. The ${ }^{1} \mathrm{H}$ NMR analysis of compounds $2 \mathrm{a}-\mathbf{e}$ and 3a-e showed that methylene hydrogens were present and simple, with displacements between 2.33 and 2.43 ppm (see Supplementary Materials). The $\mathrm{CH}_{2}$ hydrogens presented as simple with chemical shifts between 5.04 and $5.10 \mathrm{ppm}$. The hydrogens of the triazole ring characteristic of these compounds were observed as singlets in the region of 8.02 to 8.89 ppm (see Supplementary Materials).

\subsection{In Vitro Biological Evaluation}

Biological evaluations of compounds 2a-e and 3a-e were performed in K562, A549 and WSS-1 cells. As previously described, K562 is a CML cell line, A549 is a human pulmonary carcinoma epithelial cell line, and WSS-1 is a healthy human cell line. WSS-1 cells were used as a reference for the calculation of the selectivity index (SI).

\subsubsection{Cytotoxic Effects in K562 and WSS-1 Cells}

Compound screening in $\mathrm{K} 562$ cells revealed that compound $\mathbf{3 b}$ had the highest cytotoxic activity among these imatinib analogs at $10 \mu \mathrm{M}$, presenting a percentage of viability of $57.5 \%$, whereas imatinib showed $30.7 \%$ on under the same conditions. Subsequently, a concentration-response curve was carried out for compound $\mathbf{3 b}$, and an $\mathrm{IC}_{50}$ value of $35.8 \mu \mathrm{M}$ was determined (imatinib, $\mathrm{IC}_{50}=0.08 \mu \mathrm{M}$ ) (Table 1$)$. In WSS-1 cells, compound 
3b showed an $\mathrm{IC}_{50}$ of $69.3 \mu \mathrm{M}$ (imatinib, $\mathrm{IC}_{50}=9.6 \mu \mathrm{M}$ ), resulting in an SI value of 1.9 (imatinib, SI = 120) (Figure 2 and Table 1).

Table 1. Cytotoxic activity and selectivity index of imatinib and derivative $3 \mathbf{b}$ in the K562 and WSS-1 cells.

\begin{tabular}{ccccc}
\hline & & & & \\
\hline
\end{tabular}

${ }^{*} \mathrm{CI}-95 \%$ confidence interval; $\mathrm{SI}=\mathrm{IC}_{50}(\mathrm{WSS}-1) / \mathrm{IC}_{50}$ (cancer cell).

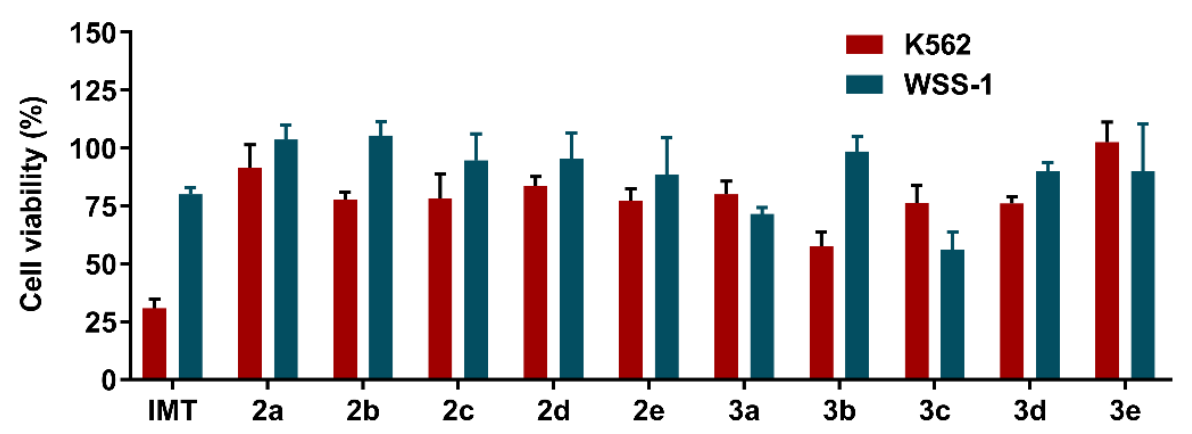

Figure 2. Screening of imatinib and its $\mathbf{2 a}-\mathbf{e}$ and $3 \mathbf{a}-\mathbf{e}$ derivatives at $10 \mu \mathrm{M}$ concentration, against human cell lines K562 (in red) and WSS-1 (green). Bars represent the mean \pm standard deviation.

Table 1 shows the $\mathrm{IC}_{50}$ and SI values of imatinib and its most cytotoxic analog, $\mathbf{3 b}$, on K562 cells.

\subsubsection{Cytotoxic Effects in A549 and WSS-1 Cells}

The evaluations performed with A549 cells showed that compounds 3a, 3c, and $3 \mathrm{~d}$ reduced cell viability by $24.6 \%, 34.0 \%$, and $49.3 \%$ at $10 \mu \mathrm{M}$, respectively (Figure 3). Subsequently, the concentration-response curves were constructed, and derivatives $\mathbf{3 a}, \mathbf{3} \mathbf{c}$, and $3 \mathrm{~d}$ exhibited $\mathrm{IC}_{50}$ values of $7.2 \mu \mathrm{M}, 6.4 \mu \mathrm{M}$, and $7.3 \mu \mathrm{M}$, respectively, proving to be approximately 10 -fold more potent than imatinib $\left(\mathrm{IC}_{50}=65.4 \mu \mathrm{M}\right)$ (Table 2).

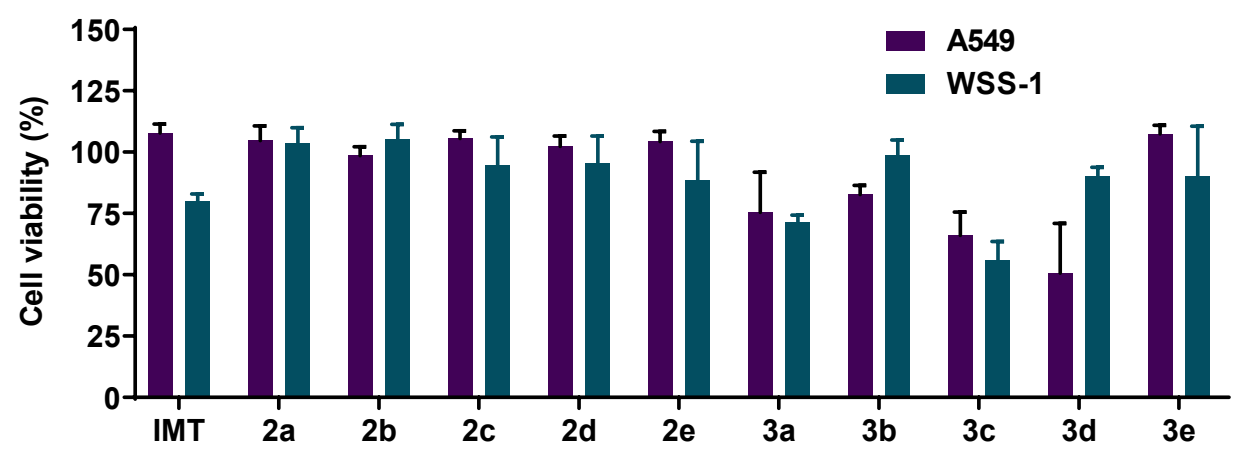

Figure 3. Screening of imatinib and its derivatives $\mathbf{2 a}-\mathbf{e}$ and $\mathbf{3 a}-\mathbf{e}$ at a concentration of $10 \mu \mathrm{M}$ against human cell lines A549 (purple) and WSS-1 (green). Bars represent the mean \pm standard deviation. 
Table 2. Cytotoxic activity and selectivity index of imatinib and derivatives $\mathbf{3 a}, \mathbf{3 c}$ and $\mathbf{3 d}$ in the A549 and WSS-1 cells.

\begin{tabular}{|c|c|c|c|c|c|}
\hline & & $\mathrm{IC}_{50}(\mu \mathrm{M})$ & & & SI \\
\hline Compounds & A549 & $\mathrm{CI}^{*}$ & WSS-1 & CI & WSS-1/A549 \\
\hline Imatinib & 65.4 & 49.5 to 86.2 & 9.6 & 8.8 to 10.4 & 0.15 \\
\hline & 7.2 & 6.1 to 8.6 & 11.6 & 8.9 to 15.6 & 1.6 \\
\hline & 6.4 & 5.3 to 7.7 & 13.5 & 12.0 to 15.1 & 2.1 \\
\hline & 7.3 & 6.3 to 8.5 & 18.6 & 13.6 to 25.5 & 2.5 \\
\hline
\end{tabular}

*CI-95\% confidence interval; $\mathrm{SI}=\mathrm{IC}_{50}$ (WSS-1) / $\mathrm{IC}_{50}$ (cancer cell).

In WSS-1 cells, 3a, 3c, and 3d showed $\mathrm{IC}_{50}$ values of 11.6, 13.5 and $18.6 \mu \mathrm{M}$ (imatinib, $\left.\mathrm{IC}_{50}=9.6 \mu \mathrm{M}\right)$ and SI values of 1.6, 2.1 and 2.5, respectively, and were up to 25-fold more selective than imatinib (SI = 0.1). Thus, in A549 cells, the new compounds $\mathbf{3 a}, \mathbf{3 c}$ and $\mathbf{3 d}$ were more potent and selective than imatinib (Figure 3 and Table 2). Imatinib was used as a standard, even though it is not a drug used to treat lung cancer. This was due to the good results obtained by Shijie and coworkers, which decreased A549 cells viability to $38.8 \%$ at $150 \mu \mathrm{M}$ (Figure 1) [18]. In addition, the literature states that imatinib can be used as a potential treatment for NSCLC, as it was able to inhibit the growth of A549 cells with an $\mathrm{IC}_{50}$ value in the range of $2-3 \mu \mathrm{M}$ [20].

Table 2 shows the $\mathrm{IC}_{50}$ and SI values of imatinib and its most cytotoxic analogs (3a, 3c and 3d) in A549 cells.

The study of the relationship between the structure and the biological activity of the synthesized compounds showed the importance of the $\mathrm{CF}_{2}$ group since, in compounds $2 \mathbf{a}-\mathbf{e}$, the absence of this group showed a loss of activity. In addition, according to the biological tests performed, the intrinsic characteristics of the substituents on the C-5 carbon of the isatin-derived ring do not seem to influence the level of activity.

\subsection{Kinase Inhibition Assay}

The compounds $\mathbf{2 a - e}$ and $\mathbf{3 a - e}$ did not show ABL1-inhibitory activity at 0.5 or $10 \mu \mathrm{M}$ under the given assay conditions. A possible explanation for this result is that the compounds may have a different mechanism of action from imatinib, which showed a subse- 
quent percentage inhibition at 0.5 and $10 \mu \mathrm{M}$ in the same assay. Another hypothesis could be a probable higher affinity of the substrate to the enzyme than the analogs, which may have interfered with the interaction of the latter with this kinase. Thus, more experiments are needed to characterize the mechanism of these compounds, by varying the enzyme inhibition assay conditions.

\section{Materials and Methods}

\subsection{Chemistry}

All reagents and solvents used were of analytical grade. Briefly, ${ }^{1} \mathrm{H},{ }^{13} \mathrm{C}$ and ${ }^{19} \mathrm{~F}$ nuclear magnetic resonance (NMR) spectra were generated at 400.00, 100.00 and 376.00 $\mathrm{MHz}$, respectively, at $25{ }^{\circ} \mathrm{C}$ using a Bruker Avance III HD instrument (Bruker AG, Fällanden, Switzerland) equipped with a prodigy BBO 400 S1 probe (Bruker AG, Fällanden, Switzerland). Tetramethylsilane was used as an internal standard. The chemical shifts $(\delta)$ are reported in ppm, and the coupling constants $(J)$ are reported in Hertz. Fourier transform infrared (FTIR) absorption spectra were recorded on a Thermo Scientific spectrophotometer (Nicolet 6700, Thermo Fisher Scientific, Waltham, MA, USA). The melting point (m.p.) values were determined using a Büchi model B-545 apparatus (Büchi Corporation, Flawil, Switzerland). Thin-layer chromatography (TLC) was performed using Merck TLC silica gel 60 F254 aluminum sheets (Merck KGaA, Darmstadt, Germany), $20 \times 20 \mathrm{~cm}$ (eluent hexane/ethyl acetate 2:8). A mass spectrometry system was coupled to obtain electron impact gas chromatography (MS-GC) spectra at $70 \mathrm{eV}$ on an Agilent 6890 apparatus, with an Agilent 5973 mass spectrometer (Agilent, Santa Clara, CA, USA). Low-resolution mass spectra were obtained by electrospray ionization (MS-ESI) on a Micromass ZQ4000 apparatus (Waters, Milford, MA, USA). High-resolution mass spectra (HR-MS) were registered using electron ionization mass spectrometry (EI-MS, digitalizing ES + capillary) on a QTOF Compact (Brucker AG, Fällanden, Switzerland).

3.1.1. General Procedure for the Preparation of 1-((1-(4-methyl-3-((4-(pyridin-3-yl) pyrimidin-2-yl)amine)phenyl)-1H-1,2,3-triazol-4-yl)methyl)indolin-2,3-dione (2a-e)

Briefly, $2.3 \mathrm{mmol}$ (1.3 eq) of the corresponding acetylenes, 12a-e, $1.8 \mathrm{mmol}(1 \mathrm{eq})$ of the $N$-(5-azido-2-methylphenyl)-4-(pyridin-3-yl)pyrimidin-2-amine (10), $0.1 \mathrm{mmol}$ (1 eq) of sodium ascorbate $0.1 \mathrm{mmol}(0.06 \mathrm{eq})$ of copper sulfate, and $20 \mathrm{~mL}$ of THF and water (1:1) were added to a monotubulated flask. The reaction was kept under magnetic stirring at room temperature for $3 \mathrm{~h}$. The progress of the reaction was monitored using TLC (hexane/ethylacetate 3:7). On completion, $30 \mathrm{~mL}$ of water was added and the mixture was extracted with $\mathrm{CHCl}_{3}(3 \times 50 \mathrm{~mL})$. The organic phase was dried with anhydrous $\mathrm{Na}_{2} \mathrm{SO}_{4}$, and the solvent was removed by evaporation. The residual crude product was purified via silica gel column chromatography, using the gradient mixture of chloroform: methanol (9.5:0.5).

The attributes can be summarized as follows:

1-((1-(4-methyl-3-((4-(pyridin-3-yl)pyrimidin-2-yl)amino)phenyl)-1H-1,2,3-triazol4-yl)methyl)indoline-2,3-dione (2a): Yield: 58\%; m.p.: 237-239 ${ }^{\circ} \mathrm{C}$. IR ( $\left.\mathrm{cm}^{-1}\right)$ : 1739; 1618; 1578; 755; 709; 656. MS-ESI [M+Na] ${ }^{+}(\%): 511$ (100). ${ }^{1} \mathrm{H}-\mathrm{NMR}\left(400 \mathrm{MHz}, \mathrm{CD}_{3} \mathrm{COCD}_{3}, \delta\right.$, ppm): 2.33 (s, 3H, H-26); 5.07 (s, 2H, H-8); 7.13 (td, J = 0.7Hz, 1H, H-6); 7.20 (d, J = 7.9 Hz, $1 \mathrm{H}, \mathrm{H}-7) ; 7.42$ (d, J = 8.5 Hz, 1H, H-14); 7.51 (m, 3H, H-13, H-19, H24); 7.58 (dd, J = 0.8 Hz, $1 \mathrm{H}, \mathrm{H}-5) ; 7.62(\mathrm{td}, J=1.3 \mathrm{~Hz}, 1 \mathrm{H}, \mathrm{H}-4) ; 8.28(\mathrm{~d}, \mathrm{~J}=2.2 \mathrm{~Hz}, 1 \mathrm{H}, \mathrm{H}-12) ; 8.47$ (dt, Jo = 8.4 Hz, $J m=2.0 \mathrm{~Hz}, 1 \mathrm{H}, \mathrm{H}-25) ; 8.56(\mathrm{~d}, J=5.2 \mathrm{~Hz}, 1 \mathrm{H}, \mathrm{H}-18) ; 8.69$ (dd, $J=1.6 \mathrm{~Hz}, 1 \mathrm{H}, \mathrm{H}-23) ; 8.82$ (s, $1 \mathrm{H}, \mathrm{H}-10) ; 9.13$ (s, 1H, NH); 9.27 (d, J = 1.6 Hz, 1H, H-22). ${ }^{13} \mathrm{C}-\mathrm{NMR}\left(100 \mathrm{MHz}, \mathrm{CD}_{3} \mathrm{COCD}_{3}\right.$, $\delta$, ppm):17.7; 34.9; 108.2; 110.9; 114.9; 115.4; 117.6; 121.5; 123.3; 123.7; 124.3; 131.2; 131.9; 134.3; 134.4; 137.9; 138.9; 142.6; 147.9; 149.9; 151.3; 157.7; 159.5; 160.6; 161.5; 182.9. HR-MS (IES) $^{+}$: Calcd for $\mathrm{C}_{27} \mathrm{H}_{20} \mathrm{~N}_{8} \mathrm{O}_{2}: 488.1709$, found: 489.1782 . Elemental analysis $\mathrm{CHN}$ : Calcd for $\mathrm{C}_{27} \mathrm{H}_{20} \mathrm{~N}_{8} \mathrm{O}_{2}$ (\%): C, 66.38; $\mathrm{H}, 4.13 ; \mathrm{N}, 22.94$, found (\%): C, 66.56; H, 4.45; N, 22.82 .

5-methyl-1-((1-(4-methyl-3-((4-(pyridin-3-yl)pyrimidin-2-yl)amino)phenyl)-1H-1,2,3triazol-4 yl)methyl)indoline-2,3-dione(2b): Yield: 41\%; m.p.: $235-237^{\circ} \mathrm{C}$. IR $\left(\mathrm{cm}^{-1}\right): 1736$; 
1580; 1532; 789; 658. MS-ESI [M-H] ${ }^{+}(\%): 501$ (100). ${ }^{1} \mathrm{H}-\mathrm{NMR}\left(400 \mathrm{MHz}, \mathrm{CD}_{3} \mathrm{COCD}_{3}, \delta\right.$, ppm): 2.26 (s, 3H, $\left.\mathrm{CH}_{3}-5\right) ; 2.33$ (s, 3H, H-26); 5.04 (s, 2H, H-8); 7.08 (d, J = 8.0 Hz, 1H, H-7); 7.41 (m, 3H, H-13, H-14, H-19); 7.53 (m, 3H, H-4, H-6, H-24); 8.28 (d, J = 2.2 Hz, 1H, H-12); $8.50(\mathrm{dt}, J o=8.1 \mathrm{~Hz}, J m=1.8 \mathrm{~Hz}, 1 \mathrm{H}, \mathrm{H}-25) ; 8.56(\mathrm{~d}, J=5.2 \mathrm{~Hz}, 1 \mathrm{H}, \mathrm{H}-18) ; 8.71(\mathrm{~d}, J=2.4 \mathrm{~Hz}$, $1 \mathrm{H}, \mathrm{H}-23) ; 8.81$ (s, 1H, H-10); 9.15 (s, 1H, NH); 9.28 (s, 1H, H-22). ${ }^{13} \mathrm{C}-\mathrm{NMR}(100 \mathrm{MHz}$, $\mathrm{CD}_{3} \mathrm{COCD}_{3}, \delta$, ppm): 17.7; 19.9; 34.9; 108.2; 110.8; 114.9; 115.4; 117.5; 121.4; 123.9; 124.6; 131.2; 131.9; 132.6; 134.4; 134.7; 138.3; 138.9; 142.6; 147.6; 147.8; 150.9; 157.8; 159.5; 160.6; 161.3; 183.2. HR-MS (IES) ${ }^{+}$: Calcd for $\mathrm{C}_{28} \mathrm{H}_{22} \mathrm{~N}_{8} \mathrm{O}_{2}$ : 502.1866, found: 503.1938. Elemental analysis CHN: Calcd for $\mathrm{C}_{28} \mathrm{H}_{22} \mathrm{~N}_{8} \mathrm{O}_{2}$ : C. 66.92; H. 4.41; N. 22.30, found (\%): C. 66.31; H. 4.50 ; N. 22.64.

5-chloro-1-((1-(4-methyl-3-((4-(pyridin-3-yl)pyrimidin-2-yl)amino)phenyl)-1H-1,2,3triazol-4 yl)methyl)indoline-2,3-dione(2c): Yield: 50\%; m.p.: $257-260{ }^{\circ} \mathrm{C}$. IR $\left(\mathrm{cm}^{-1}\right)$ : 1739 ; 1579; 1531; 809; 656. MS-ESI [M-H] ${ }^{-}$(\%): 521 (100), ${ }^{1} \mathrm{H}-\mathrm{NMR}\left(400 \mathrm{MHz}, \mathrm{CD}_{3} \mathrm{COCD}_{3}, \delta\right.$, ppm): 2.33 (s, 3H, H-26); 5.07 (s, 2H, H-8); 7.23 (d, J = 8.4 Hz, 1H, H-7); 7.42 (d, J = 8.4 Hz, 1H, H-6); 7.51 (m, 2H, H-13, H-19); 7.55 (dd, J = 4.8 Hz, 1H, H-24); 7.66 (m, 2H, H-4, $\mathrm{H}-14) ; 8.27$ (d, J = 2.2 Hz, 1H, H-12); 8.50 (dt, Jo = 8.1 Hz, Jm = 1.6 Hz,. 1H, H-25); 8.56 (d, $J=5.2 \mathrm{~Hz}, 1 \mathrm{H}, \mathrm{H}-18) ; 8.71$ (s, 1H, H-23); 8.75 (s, 1H, H-10); 9.15 (s, 1H, NH); 9.28 (s, 1H, $\mathrm{H}-22) .{ }^{13} \mathrm{C}-\mathrm{NMR}\left(100 \mathrm{MHz}, \mathrm{CD}_{3} \mathrm{COCD}_{3}, \delta, \mathrm{ppm}\right): 17.7 ; 35.1 ; 108.2 ; 112.7 ; 114.9 ; 115.4 ; 119.0$; $121.5 ; 123.8 ; 123.9 ; 127.5 ; 131.3 ; 131.9 ; 134.4 ; 134.8 ; 136.8 ; 138.9 ; 142.4 ; 147.5 ; 148.4 ; 150.9$; 157.5; 159.5; 160.6; 161.3; 181.8. HR-MS (IES) ${ }^{+}$: Calcd for $\mathrm{C}_{27} \mathrm{H}_{19} \mathrm{ClN}_{8} \mathrm{O}_{2}$ : 522.1319, found: 523.1392. Elemental analysis $\mathrm{CHN}$ : Calcd for $\mathrm{C}_{27} \mathrm{H}_{19} \mathrm{ClN}_{8} \mathrm{O}_{2}$ : C. 62.01; H. 3.66; N. 21.43, found (\%): C. $62.31 ;$ H. 3.14; N. 21.65.

5-bromo-1-((1-(4-methyl-3-((4-(pyridin-3-yl)pyrimidin-2-yl)amino)phenyl)-1H-1,2,3triazol-4 yl)methyl)indoline-2,3-dione (2d): Yield: 50\%; m.p.: $267-270{ }^{\circ} \mathrm{C}$. IR $\left(\mathrm{cm}^{-1}\right): 1736$; 1579; 1531; 808; 653. MS-ESI [M+Na] ${ }^{+}(\%): 589$ (80). ${ }^{1} \mathrm{H}-\mathrm{NMR}\left(400 \mathrm{MHz}, \mathrm{CD}_{3} \mathrm{COCD}_{3}, \delta\right.$, ppm): 2.33 (s, 3H, H-26); 5.07 (s, 2H, H-8); 7.18 (d, J = 8.4 Hz, 1H, H-7); 7.42 (d, J = 8.4 Hz, 1H, H-6); 7.51 (m, 2H, H-13, H-19); 7.56 (dd, J = 5.0 Hz, 1H, H-24); 7.74 (d, J= 2.1 Hz, 1H, $\mathrm{H}-14) ; 7.80(\mathrm{dd}, J=2.1 \mathrm{~Hz}, 1 \mathrm{H}, \mathrm{H}-4) ; 8.27$ (d, J = 2.2 Hz, 1H, H-12); 8.51 (dt, Jo = $8.0 \mathrm{~Hz}$, $J m=1.8 \mathrm{~Hz}, 1 \mathrm{H}, \mathrm{H}-25) ; 8.56$ (d, $J=5.2 \mathrm{~Hz}, 1 \mathrm{H}, \mathrm{H}-18) ; 8.71$ (dd, $J=1.4 \mathrm{~Hz}, 1 \mathrm{H}, \mathrm{H}-23) ; 8.80$ (s, $1 \mathrm{H}, \mathrm{H}-10) ; 9.15$ (s, 1H, NH); 9.2 (d, $J=1.56 \mathrm{~Hz}, 1 \mathrm{H}, \mathrm{H}-22) .{ }^{13} \mathrm{C}-\mathrm{NMR}\left(100 \mathrm{MHz}, \mathrm{CD}_{3} \mathrm{COCD}_{3}\right.$, $\delta$, ppm): 17.7; 35.0; 108.2; 113.1; 115.0; 115.4; 119.0; 121.5; 123.9; 126.6; 131.3; 131.9; 132.1; $134.4 ; 134.9 ; 138.9 ; 139.6 ; 142.4 ; 147.5 ; 148.8 ; 150.8 ; 157.3 ; 159.5 ; 160.6 ; 161.3 ; 181.7$. HR-MS (IES) $^{+}$: Calcd for $\mathrm{C}_{27} \mathrm{H}_{19} \mathrm{BrN}_{8} \mathrm{O}_{2}$ : 566.0814, found: 567.0887. Elemental analysis CHN: Calcd for $\mathrm{C}_{27} \mathrm{H}_{19} \mathrm{BrN}_{8} \mathrm{O}_{2}$ : C. 57.15; H. 3.38; N. 19.75, found (\%): C. 57.00; H. 3.21; N. 19.77.

5-fluoro-1-((1-(4-methyl-3-((4-(pyridin-3-yl)pyrimidin-2-yl)amino)phenyl)-1H-1,2,3triazol-4 yl)methyl)indoline-2,3-dione (2e): Yield: 82\%; m.p.: 251-253 ${ }^{\circ} \mathrm{C}$. IR $\left(\mathrm{cm}^{-1}\right)$ : 1736 ; 1581; 1537; 809. MS-ESI [M-H] ${ }^{+}$(\%): 505 (70). ${ }^{1} \mathrm{H}-\mathrm{NMR}\left(400 \mathrm{MHz}, \mathrm{CD}_{3} \mathrm{COCD}_{3}, \delta, \mathrm{ppm}\right)$ : 2.33 (s, 3H, H-26); 5.07 (s, 2H, H-8); 7.22 (dd, J = 3.7 Hz, 1H, H-7); 7.43 (d, J = 8.4 Hz, 1H, $\mathrm{H}-14) ; 7.50$ (m, 5H, H-4, H-6, H-13, H-19, H-24); 8.27 (d, J = 2.0 Hz, 1H, H-12); 8.50 (dt, $J o=8.2 \mathrm{~Hz}, J m=1.9 \mathrm{~Hz}, 1 \mathrm{H}, \mathrm{H}-25) ; 8.55$ (d, $J=5.2 \mathrm{~Hz}, 1 \mathrm{H}, \mathrm{H}-18) ; 8.68$ (dd, J = $1.5 \mathrm{~Hz}, \mathrm{H}-23)$; 8.81 (s, 1H, H-10); 9.14 (s, 1H, NH); 9.26 (d, $J=1.7 \mathrm{~Hz}, 1 \mathrm{H}, \mathrm{H}-22) .{ }^{13} \mathrm{C}-\mathrm{NMR}(100 \mathrm{MHz}$, $\left.\mathrm{CD}_{3} \mathrm{COCD}_{3}, \delta, \mathrm{ppm}\right): 17.8 ; 35.1 ; 108.3 ; 111.5(\mathrm{~d}, J=24.4 \mathrm{~Hz}) ; 112.5(\mathrm{~d}, J=7.5 \mathrm{~Hz}) ; 115.1 ; 115.5$; 118.7 (d, $J=7.14 \mathrm{~Hz}) ; 121.6 ; 123.8 ; 124.1 ; 131.4 ; 132.0 ; 134.4 ; 134.5 ; 139.0 ; 142.6 ; 146.3 ; 148.1$; $151.5 ; 158.5$ (d, $J=239.8 \mathrm{~Hz}) ; 157.9$ (d, $J=1.0 \mathrm{~Hz}) ; 159.6 ; 160.7 ; 161.6 ; 182.4$ (d, $J=1.3 \mathrm{~Hz})$.

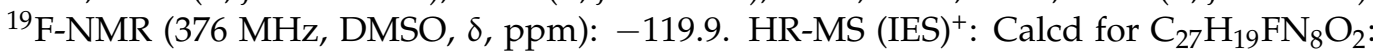
506.1615, found: 507.1687. Elemental analysis Calcd for $\mathrm{C}_{27} \mathrm{H}_{19} \mathrm{FN}_{8} \mathrm{O}_{2}$ : C. 64.03; H. 3.78; N. 22.12, found (\%): C. 64.68; H. 3.41; N. 22.62.

3.1.2. General Procedure for the Preparation of 3.3-difluoro-1-((1-(4-methyl-3-

((4-(pyridine-3-yl) pyrimidin-2-yl)amino) phenyl)-1H-1,2,3-triazole-4-yl)methyl)indoline2-one (3a-e)

Briefly, $2.3 \mathrm{mmol}(1.3 \mathrm{eq})$ of the corresponding acetylenes 14a-e, $1.8 \mathrm{mmol}(1 \mathrm{eq})$ of the $N$-(5-azido-2-methylphenyl)-4-(pyridine-3-yl)pyrimidin-2-amine (10), $0.1 \mathrm{mmol}$ (1 eq) of sodium ascorbate $0.1 \mathrm{mmol}(0.06 \mathrm{eq})$ of copper sulfate and $20 \mathrm{~mL}$ of THF and water (1:1) were added in a monotube flask. The reaction was kept under magnetic stirring at room 
temperature for $3 \mathrm{~h}$. The progress of the reaction was monitored using TLC (hexane/ethyl acetate 3:7). On completion, $30 \mathrm{~mL}$ of water was added, and the mixture was extracted with $\mathrm{CHCl}_{3}(3 \times 50 \mathrm{~mL})$. The organic phase was dried with anhydrous $\mathrm{Na}_{2} \mathrm{SO}_{4}$, and the solvent was removed by evaporation. The residual crude product was purified via silica gel column chromatography using the gradient mixture chloroform: methanol (9.5:0.5).

The analyses can be summarized as follows:

3.3-difluoro-1-((1-(4-methyl-3-((4-(pyridin-3-yl)pyrimidin-2-yl)amino)phenyl)-1H1,2,3-triazole-4-yl)methyl)indoline-2-one (3a): Yield: 51\%; m.p.: $222{ }^{\circ} \mathrm{C}$. IR $\left(\mathrm{cm}^{-1}\right): 3435$; 3123; 1746; 1580; 1286. MS-ESI [M+1] $]^{+}(\%): 511$ (100). ${ }^{1} \mathrm{H}-\mathrm{NMR}\left(400 \mathrm{MHz}, \mathrm{CD}_{3} \mathrm{COCD}_{3}, \delta\right.$, ppm): 2.34 (s, 3H, H-26); 5.09 (s, 2H, H-8); 7.24 (t, J = 7.6 Hz, 1H, H-6); 7.34 (d, J = 7.9 Hz, $1 \mathrm{H}, \mathrm{H}-7) ; 7.44$ (d, J = 8.4 Hz, 1H, H-14); 7.50 (m, 2H, H-19, H-24); 7.58 (m, 2H, H-5, H13); $7.72(\mathrm{~d}, J=7.4 \mathrm{~Hz}, 1 \mathrm{H}, \mathrm{H}-4) ; 8.31(\mathrm{~d}, J=2.2 \mathrm{~Hz}, 1 \mathrm{H}, \mathrm{H}-12) ; 8.47$ (dt, Jo = 8.1 Hz, $J m=1.6 \mathrm{~Hz}, 1 \mathrm{H}, \mathrm{H}-25) ; 8.57$ (d, J = 5.2 Hz, 1H, H-18); 8.70 (s, H, H-23); 8.89 (s, 1H, H-10); 9.15 (s, 1H, NH); 9.29 (s, 1H, H-22). ${ }^{13} \mathrm{C}-\mathrm{NMR}\left(100 \mathrm{MHz}, \mathrm{CD}_{3} \mathrm{COCD}_{3}, \delta, \mathrm{ppm}\right): 17.8 ; 35.2$; 108.3; $111.0(\mathrm{t}, \mathrm{J}=246.6 \mathrm{~Hz}) ; 111.4 ; 115.3 ; 115.7 ; 118.7(\mathrm{t}, \mathrm{J}=22.7 \mathrm{~Hz}) ; 121.9 ; 123.8 ; 124.0$; 124.6; 131.3; 132.0; 134.2; 134.4; 139.0; 141.8; 142.9 (t, J = 7.0 Hz); 148.0; 151.4; 159.6; 160.7; 161.6; $164.0(\mathrm{t}, J=29.7 \mathrm{~Hz}) .{ }^{19} \mathrm{~F}-\mathrm{NMR}(376 \mathrm{MHz}, \mathrm{DMSO}, \delta, \mathrm{ppm}):-110.4$. HR-MS (IES) ${ }^{+}$: Calcd for $\mathrm{C}_{27} \mathrm{H}_{20} \mathrm{~F}_{2} \mathrm{~N}_{8} \mathrm{O}$ : 510.1728, found: 511.1800. Elemental analysis CHN: Calcd for $\mathrm{C}_{27} \mathrm{H}_{20} \mathrm{~F}_{2} \mathrm{~N}_{8} \mathrm{O}$ : C. 63.52; H. 3.95; N. 21.95, found (\%): C. 63.35; H. 3.81; N. 21.68.

3,3-difluoro-5-methyl-1-((1-(4-methyl-3-((4-(pyridin-3-yl)pyrimidin-2-yl)amino)phenyl)1H-1,2,3-triazol-4-yl)methyl)indolin-2-one (3b): Yield: $25 \%$; m.p.: $102-104{ }^{\circ} \mathrm{C} . \mathrm{IR}\left(\mathrm{cm}^{-1}\right)$ : 1746; 1578; 1298. MS-ESI [M+Na] ${ }^{+}(\%): 547(100) .{ }^{1} \mathrm{H}-\mathrm{NMR}\left(400 \mathrm{MHz}, \mathrm{CD}_{3} \mathrm{COCD}_{3}, \delta, \mathrm{ppm}\right)$ : 2.34 (s, 3H, H-26); 2.42 (s, 3H, H-5'); 5.07 (s, 2H, H-8); 7.16 (s, 1H, NH); 7.26 (m, 4H, H-6, $\mathrm{H}-7, \mathrm{H}-14) ; 7.29$ (m, 2H, H-19, H-24); 7.35 (s, 1H, H-13); 7.48 (dd, J = 4.8 Hz, 1H, H-4); 8.02 (s, $1 \mathrm{H}, \mathrm{H}-10) ; 8.47$ (dt, Jo = 8.4 Hz, Jm = $1.8 \mathrm{~Hz}, 1 \mathrm{H}, \mathrm{H}-25) ; 8.55(\mathrm{~d}, J=5.2 \mathrm{~Hz}, 1 \mathrm{H}, \mathrm{H}-18) ; 8.74(\mathrm{~d}$, $J=3.6 \mathrm{~Hz}, 1 \mathrm{H}, \mathrm{H}-23) ; 8.94(\mathrm{~d}, J=2.0 \mathrm{~Hz}, 1 \mathrm{H}, \mathrm{H}-12) ; 9.26$ (d, $J=1.0 \mathrm{~Hz}, 1 \mathrm{H}, \mathrm{H}-22) .{ }^{13} \mathrm{C}-\mathrm{NMR}$ (100 MHz, $\left.\mathrm{CD}_{3} \mathrm{COCD}_{3}, \delta, \mathrm{ppm}\right): 17.8 ; 20.9 ; 35.6 ; 109.0 ; 111.1(\mathrm{t}, J=248.4 \mathrm{~Hz}) ; 110.9 ; 112.3$; $114.2 ; 119.9(\mathrm{t}, J=22.6 \mathrm{~Hz}) ; 120.9 ; 123.9 ; 125.2 ; 127.4 ; 131.3 ; 132.4 ; 133.9 ; 134.1 ; 138.7 ; 140.1(\mathrm{t}$, $J=7.1 \mathrm{~Hz}) ; 142.2 ; 148.4 ; 151.8 ; 159.2 ; 160.1 ; 162.7 ; 165.2(\mathrm{t}, J=30.6 \mathrm{~Hz}) .{ }^{19} \mathrm{~F}-\mathrm{NMR}(376 \mathrm{MHz}$, DMSO, $\delta, p p m):-111.6$. HR-MS (IES) ${ }^{+}$: Calcd for $\mathrm{C}_{28} \mathrm{H}_{22} \mathrm{~F}_{2} \mathrm{~N}_{8} \mathrm{O}: 524.1885$, found: 525.1957. Elemental analysis CHN: Calcd for $\mathrm{C}_{28} \mathrm{H}_{22} \mathrm{~F}_{2} \mathrm{~N}_{8}$ O: C. 64.12; H. 4.23; N. 21.36, found (\%): C. 64.27 ; H. 3.99; N. 21.01.

5-chloro-3,3-difluoro-1-((1-(4-methyl-3-((4-(pyridin-3-yl)pyrimidin-2-yl)amino)phenyl)1H-1,2,3-triazol-4-yl)methyl)indoline-2-one (3c): Yield: $45 \%$; m.p.: $223-224{ }^{\circ} \mathrm{C}$. IR $\left(\mathrm{cm}^{-1}\right)$ : 3435; 3123; 1754; 1580; 1286. MS-ESI $[\mathrm{M}+\mathrm{Na}]^{+}(\%): 567$ (100). ${ }^{1} \mathrm{H}-\mathrm{NMR}(400 \mathrm{MHz}$, $\left.\mathrm{CD}_{3} \mathrm{COCD}_{3}, \delta, \mathrm{ppm}\right): 2.34$ (s, 3H, H-26); 5.10 (s, 2H, H-8); 7.37 (d, J = 8.5 Hz, 1H, H-7); 7.44 $(\mathrm{d}, J=8.4 \mathrm{~Hz}, 1 \mathrm{H}, \mathrm{H}-14) ; 7.55$ (m, 3H, H-13, H-19, H-24); 7.66 (d, J = 8.4 Hz, 1H, H-6); 7.94 (d, $J=1.8 \mathrm{~Hz}, 1 \mathrm{H}, \mathrm{H}-4) ; 8.30$ (d, $J=2.1 \mathrm{~Hz}, 1 \mathrm{H}, \mathrm{H}-12) ; 8.47$ (d, J = $7.9 \mathrm{~Hz}, 1 \mathrm{H}, \mathrm{H}-25) ; 8.57$ (d, J= 5.2 Hz, 1H, H-18); 8.72 (s, 1H, H-23); 8.87 (s, 1H, H-10); 9.15 (s, 1H, NH); 9.31 (s, 1H, $\mathrm{H}-22) .{ }^{13} \mathrm{C}-\mathrm{NMR}\left(100 \mathrm{MHz}, \mathrm{CD}_{3} \mathrm{COCD}_{3}, \delta, \mathrm{ppm}\right): 17.7 ; 35.3 ; 108.2 ; 110.3(\mathrm{t}, J=248.1 \mathrm{~Hz})$; $113.0 ; 115.2 ; 115.6 ; 120.3(\mathrm{t}, J=22.7 \mathrm{~Hz}) ; 121.8 ; 124.9 ; 128.1 ; 131.2 ; 131.9 ; 133.9 ; 134.3 ; 134.4 ;$ $138.9 ; 141.5 ; 141.7(\mathrm{t}, J=6.7 \mathrm{~Hz}) ; 147.9 ; 151.2 ; 159.5 ; 160.6 ; 161.5 ; 163.7(\mathrm{t}, J=29.6 \mathrm{~Hz})$. ${ }^{19}$ F-NMR (376 MHz, DMSO, $\delta$, ppm): -111.7. HR-MS (IES) ${ }^{+}$: Calcd for $\mathrm{C}_{27} \mathrm{H}_{19} \mathrm{ClF}_{2} \mathrm{~N}_{8} \mathrm{O}$ : 544.1338, found: 545.1411. Elemental analysis $\mathrm{CHN}$ : Calcd for $\mathrm{C}_{27} \mathrm{H}_{19} \mathrm{ClF}_{2} \mathrm{~N}_{8} \mathrm{O}: \mathrm{C}, 59.51 ; \mathrm{H}$, $3.51 ; \mathrm{N}, 20.56$, found (\%): C, 59.35; H, 3.35; N, 20.23.

5-bromo-3.3-difluoro-1-((1-(4-methyl-3-((4-(pyridin-3-yl)pyrimidin-2-yl)amino)phenyl)1H-1,2,3-triazol-4-yl)methyl)indoline-2-one (3d):Yield: $78 \%$; m.p.: $218-219{ }^{\circ} \mathrm{C}$. IR $\left(\mathrm{cm}^{-1}\right)$ : 3056; 1753; 1579; 1284. MS-ESI [M+1] ${ }^{+}$(\%): 590 (100). ${ }^{1} \mathrm{H}-\mathrm{NMR}\left(400 \mathrm{MHz}, \mathrm{CD}_{3} \mathrm{COCD}_{3}, \delta\right.$, ppm): 2.34 (s, 3H, H-26); 5.09 (s, 2H, H-8); 7.30 (d, J = 8.5 Hz, 1H, H-7); 7.44 (d, J = 8.4 Hz, 1H, $\mathrm{H}-14) ; 7.51$ (m, 2H, H-19, H-24); 7.55 (dd, J = 2.3 Hz, 1H, H-13); 7.80 (d, J = $8.5 \mathrm{~Hz}, 1 \mathrm{H}, \mathrm{H}-6)$; $8.04(\mathrm{~d}, J=1.7 \mathrm{~Hz}, 1 \mathrm{H}, \mathrm{H}-4) ; 8.30$ (d, $J=2.2 \mathrm{~Hz}, 1 \mathrm{H}, \mathrm{H}-12) ; 8.46$ (d, $J=8.0 \mathrm{~Hz}, 1 \mathrm{H}, \mathrm{H}-25) ; 8.57$ (d, $J=5.2 \mathrm{~Hz}, 1 \mathrm{H}, \mathrm{H}-18) ; 8.70$ (s, 1H, H-23); 8.87 (s,1H, H-10); 9.15 (s, 1H, NH); 9.29 (s, 1H, $\mathrm{H}-22) .{ }^{13} \mathrm{C}-\mathrm{NMR}\left(100 \mathrm{MHz}, \mathrm{CD}_{3} \mathrm{COCD}_{3}, \delta, \mathrm{ppm}\right): 17.7 ; 35.2 ; 108.2 ; 110.2(\mathrm{t}, J=248.1 \mathrm{~Hz})$; $113.4 ; 115.2 ; 115.5 ; 115.7 ; 120.6(\mathrm{t}, J=22.8 \mathrm{~Hz}) ; 121.8 ; 123.7 ; 127.5 ; 131.2 ; 131.9 ; 134.4 ; 136.8$; 
$138.9 ; 141.5 ; 142.2(\mathrm{t}, J=6.8 \mathrm{~Hz}) ; 148.0 ; 151.4 ; 159.5 ; 160.6 ; 161.5 ; 163.5(\mathrm{t}, J=29.8 \mathrm{~Hz})$.

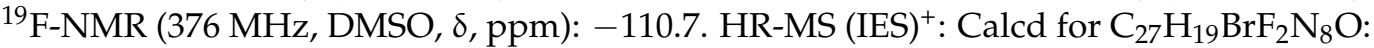
588.0833, found: 589.0906. Elemental analysis $\mathrm{CHN}$ : Calcd for $\mathrm{C}_{27} \mathrm{H}_{19} \mathrm{BrF}_{2} \mathrm{~N}_{8} \mathrm{O}$ : C, 55.02; $\mathrm{H}$, 3.25; N, 19.01, found (\%): C, 54.97; H, 3.19; N, 19.60 .

(4-methyl-3-((4-(pyridin-3-yl)pyrimidin-2-yl)amino)phenyl)-1H-1,2,3-triazol-4-yl) methyl)indoline-2-one (3e): Yield: 30\%; m.p.: $220^{\circ} \mathrm{C}, \mathrm{IR}\left(\mathrm{cm}^{-1}\right)$ : 3737; 3055; 1748; 1578; 1291. MS-ESI [M+1] ${ }^{+}(\%): 529$ (100). ${ }^{1} \mathrm{H}-\mathrm{NMR}$ (400 MHz, $\left.\mathrm{CD}_{3} \mathrm{COCD}_{3}, \delta, \mathrm{ppm}\right): 2.43$ (s, 3H, $\mathrm{H}-26) ; 5.07$ (s, 2H, H-8); 7.15 (s, 1H, NH); 7.21 (m, 1H, H-7); 7.27 (m, 2H, H-14, H-24); 7.31 (m, 2H, H-13, H-19); 7.40 (dd, $J=3.8 \mathrm{~Hz}, 1 \mathrm{H}, \mathrm{H}-6) ; 7.49$ (dd, $J=4.8 \mathrm{~Hz}, 1 \mathrm{H}, \mathrm{H}-4) ; 8.05$ (s, 1H, $\mathrm{H}-10) ; 8.47(\mathrm{dt}, J o=8.1 \mathrm{~Hz}, J m=1.9 \mathrm{~Hz}, 1 \mathrm{H}, \mathrm{H}-25) ; 8.56(\mathrm{~d}, J=5.2 \mathrm{~Hz}, 1 \mathrm{H}, \mathrm{H}-18) ; 8.76(\mathrm{~d}$, $J=3.9 \mathrm{~Hz}, 1 \mathrm{H}, \mathrm{H}-23) ; 8.95$ (d, $J=1.9 \mathrm{~Hz}, 1 \mathrm{H}, \mathrm{NH}) ; 9.27$ (s,1H, H-22). ${ }^{13} \mathrm{C}-\mathrm{NMR}(100 \mathrm{MHz}$, $\left.\mathrm{CD}_{3} \mathrm{COCD}_{3}, \delta, \mathrm{ppm}\right): 17.8 ; 35.7 ; 109.1 ; 112.2 ; 112.5 ; 112.6(\mathrm{t}, J=25.6 \mathrm{~Hz}) ; 114.2 ; 120.3(\mathrm{~d}$, $J=23.4 \mathrm{~Hz}) ; 121.1 ; 127.5 ; 131.3 ; 132.4 ; 134.7 ; 135.3(\mathrm{t}, J=297.0 \mathrm{~Hz}) ; 138.8 ; 141.8 ; 148.5 ; 151.8$; $158.5(\mathrm{~d}, J=239.8 \mathrm{~Hz}) ; 159.2 ; 160.1 ; 162.8 ; 164.0(\mathrm{t}, J=29.7 \mathrm{~Hz}) .{ }^{19} \mathrm{~F}-\mathrm{NMR}(376 \mathrm{MHz}, \mathrm{DMSO}$, $\delta$, ppm): $-111,9,-117,1$. HR-MS (IES) ${ }^{+}$: Calcd for $\mathrm{C}_{27} \mathrm{H}_{19} \mathrm{~F}_{3} \mathrm{~N}_{8} \mathrm{O}: 528.1634$, found: 529.1706 . Elemental analysis $\mathrm{CHN}$ : Calcd for $\mathrm{C}_{27} \mathrm{H}_{19} \mathrm{~F}_{3} \mathrm{~N}_{8} \mathrm{O}$ : C, 61.36; H, 3.62; N, 21.20, found (\%): C, $61.19 ; \mathrm{H}, 3.39 ; \mathrm{N}, 21.15$.

3.1.3. General Procedure for the Preparation of N-(5-azido-2-methylphenyl)-4-(pyridin-3-yl) pyrimidin-2-amine (10)

Briefly, 2-phenylaminopyrimidine (PAPP) (9) (1 eq) was added in $30 \mathrm{~mL}$ of water in a batch-flask under an ice bath; sulfuric acid was then added until the suspension became a solution. At this point, the sodium nitrites $(1.5 \mathrm{eq})$ in $2 \mathrm{~mL}$ of water were added to the mixture, which was kept under magnetic stirring for $30 \mathrm{~min}$, at -5 to $0{ }^{\circ} \mathrm{C}$. Subsequently, sodium azide ( $2 \mathrm{eq}$ ) in $2 \mathrm{~mL}$ of water was added, and the mixture was kept under magnetic stirring for $10 \mathrm{~min}$ at -5 to $0{ }^{\circ} \mathrm{C}$. Thereafter, the ice bath was removed, and the medium was left under magnetic stirring at room temperature for $3 \mathrm{~h}$. The progress of the reaction was monitored using TLC (chloroform/methanol 9:1). On completion, the $\mathrm{pH}$ was adjusted to 7.0 with potassium carbonate solution and then extracted with dichloromethane $(3 \times 50 \mathrm{~mL})$. The organic phase was dried with anhydrous $\mathrm{Na}_{2} \mathrm{SO}_{4}$, and the solvent was removed by evaporation. The product was obtained as a slightly yellowish solid.

Yield: $98 \%$; m.p.: $115.3{ }^{\circ} \mathrm{C} . \mathrm{IR}\left(\mathrm{cm}^{-1}\right): 3325 ; 2109 ; 2079 ; 1526 ; 790 ; 695 ; 687 . \mathrm{MS}-\mathrm{ESI}$ $[\mathrm{M}-\mathrm{H}]^{+}(\%): 302(100)$.

\subsubsection{General Procedure for the Preparation of 1-propyl-indoline-2,3-dione (12a-e)}

Briefly, $6.8 \mathrm{mmol}(1 \mathrm{eq})$ of the corresponding isatins (11a-e), $11 \mathrm{mmol}(1.63 \mathrm{eq})$ of propargyl bromide, $12.92 \mathrm{mmol}(1.9 \mathrm{eq})$ of potassium carbonate, $2.1 \mathrm{mmol}$ (0.32 eq) of sodium iodide and $6 \mathrm{~mL}$ of anhydrous DMF were added to a monotube flask. The progress of the reaction was monitored using TLC (hexane/ethylacetate 7:3). After $2 \mathrm{~h}$ at room temperature, $5 \mathrm{~mL}$ of $3 \mathrm{~N} \mathrm{HCl}$ solution and ice were added, and a precipitate was formed. This solid was filtered by vacuum to give the desired product.

The attributes can be summarized as follows:

1-propyl-indoline-2,3-dione (12a): Yield: 97\%. m.p.: $144-146{ }^{\circ} \mathrm{C}$ (lit. 144-146 ${ }^{\circ} \mathrm{C}$ ) [19]. IR $\left(\mathrm{cm}^{-1}\right)$ : 3242; 2124; 1725; 1617. MS-GC (70 eV, m/z, \%): 129 (100); 185 (81); 102 (54); 90 (48); 128 (39).

5-methyl-1-propylindoline-2,3-dione (12b): Yield: 83\%. m.p.: $147-150^{\circ} \mathrm{C}$ (lit. 149-151 $\left.{ }^{\circ} \mathrm{C}\right)$ [19]. IR ( $\left.\mathrm{cm}^{-1}\right)$ : 3243; 2126; 1725; 1616. MS-GC (70 eV, m/z, \%): 199 (100), 143 (99); 142 (80); 115 (52); 116 (27).

5-chloro-1-propylindoline-2,3-dione (12c): Yield: 90\%. m.p.: 157-159 ${ }^{\circ} \mathrm{C}$ (lit. 158-159 ${ }^{\circ} \mathrm{C}$ ) [19]. IR ( $\left.\mathrm{cm}^{-1}\right)$ : 3225; 2120; 1726; 1601. MS-GC (70 eV, $\left.\mathrm{m} / \mathrm{z}, \%\right): 163$ (100); 219 (92); 228 (68); 124 (46); 74 (51); 165 (34).

5-bromo-1-propylindoline-2,3-dione(12d): Yield: 90\%. m.p.: $161-162{ }^{\circ} \mathrm{C}$ (lit. 157-158 ${ }^{\circ} \mathrm{C}$ ) [21]. IR ( $\left.\mathrm{cm}^{-1}\right)$ : 3243; 2106; 1729; 1603. MS-GC (70 eV, m/z, \%): 128 (100); 208 (84); 206 (83), 262 (82); $264(81)$. 
5-fluoro-1-propylindoline-2,3-dione (12e): Yield: 96\%. m.p.: $148-152{ }^{\circ} \mathrm{C}$ (lit. 158-159 ${ }^{\circ} \mathrm{C}$ ) [21]. IR $\left(\mathrm{cm}^{-1}\right)$ : 3225; 2121; 1726; 1762. MS-GC (70 eV, m/z, \%): 203 (99); 147 (100); 120 (62); 108 (57); $146(41)$.

\subsubsection{General Procedure for the Preparation of 3,3-difluoroindolin-2-one (13a-e)}

Briefly, $3.4 \mathrm{mmol}$ (1 eq) of the corresponding isatins 11a-e, $17 \mathrm{mmol}$ (5 eq) of DAST and $30 \mathrm{~mL}$ of $\mathrm{CH}_{2} \mathrm{Cl}_{2}$ were added into a monotube flask. The mixture was maintained at room temperature with magnetic stirring under a nitrogen atmosphere for $4 \mathrm{~h}$. The progress of the reaction was monitored using TLC (hexane/ethyl acetate 7:3). The mixture was washed with water $(3 \times 40 \mathrm{~mL})$, then the organic phase was dried with anhydrous $\mathrm{Na}_{2} \mathrm{SO}_{4}$, and the solvent was removed by evaporation. The product was obtained as a white solid.

The analyses can be summarized as follows:

3,3-difluoroindolin-2-one (13a): Yield: 77\%; 136-138 ${ }^{\circ} \mathrm{C}$ (lit. 137-139 $\left.{ }^{\circ} \mathrm{C}\right)$ [21]. IR ( $\left.\mathrm{cm}^{-1}\right)$ : 3250; 1744; 1304. MS-ESI [M-1] ${ }^{+},(\%): 168$ (100). MS-GC (70 eV, m/z, \%): 169 (100); 141 (92); 114 (86); 75 (10); 126 (10).

3,3-difluoro-5-methylindoline-2-one (13b): Yield: 88\%; m.p.: $157-159{ }^{\circ} \mathrm{C}$ (lit. 155-157 $\left.{ }^{\circ} \mathrm{C}\right)$ [22] IR (cm $\left.{ }^{-1}\right)$ : 3247; 1747; 1331. MS-GC (70 eV, m/z, \%): 182 (100); 221 (94); 192 (17); 178 (13); 122 (13).

5-chloro-3,3-difluoroindolin-2-one (13c): Yield: 68\%; m.p.: 180-182 ${ }^{\circ} \mathrm{C}$ (lit. 183-185 ${ }^{\circ} \mathrm{C}$ ) [21]. IR ( $\left.\mathrm{cm}^{-1}\right)$ : 3243; 1726; 1335. MS-GC (70 eV, m/z, \%): 175 (100); 203 (83); 148 (60); 177 (32); 205 (27).

5-bromo-3,3-difluoroindolin-2-one (13d): Yield: 75\%; m.p.: 192-194 ${ }^{\circ} \mathrm{C}$. IR $\left(\mathrm{cm}^{-1}\right)$ :

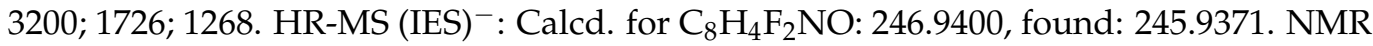
${ }^{1} \mathrm{H}\left(400 \mathrm{MHz} ; \mathrm{CD}_{3} \mathrm{COCD}_{3}, \delta, \mathrm{ppm}\right): 6.95$ (dd, J = $\left.1.5 \mathrm{~Hz}, 1 \mathrm{H}, \mathrm{H}-7\right) ; 7.71$ (m, 1H, H-6); $7.96(\mathrm{~m}, 1 \mathrm{H}, \mathrm{H}-4) ; 11.32$ (s, 1H, NH), NMR ${ }^{13} \mathrm{C}\left(100 \mathrm{MHz} ; \mathrm{CD}_{3} \mathrm{COCD}_{3}, \delta, \mathrm{ppm}\right): 110.5(\mathrm{t}$, $J=248.6 \mathrm{~Hz}, \mathrm{C}-3) ; 113.8(\mathrm{C}-7) ; 114.7(\mathrm{t}, J=2.1 \mathrm{~Hz}, \mathrm{C}-5) ; 121.0(\mathrm{t}, J=22.8 \mathrm{~Hz}, \mathrm{C}-4) ; 127.8$ (C-6); $136.8(\mathrm{C}-7 \mathrm{a}) ; 141.8(\mathrm{t}, J=7.4 \mathrm{~Hz}, \mathrm{C}-3 \mathrm{a}) ; 165.2(\mathrm{t}, J=29.1 \mathrm{~Hz}, \mathrm{C}-2), \mathrm{NMR}{ }^{19} \mathrm{~F}(376 \mathrm{MHz}$; DMSO, $\delta$, ppm): -111.0 .

5-fluoro-3,3-difluoroindolin-2-one (13e): Yield: 80\%; m.p.: $184-186{ }^{\circ} \mathrm{C} . \mathrm{IR}\left(\mathrm{cm}^{-1}\right)$ : 3271; 1739; 1282. HR-MS (IES) ${ }^{-}$: Calcd. For $\mathrm{C}_{8} \mathrm{H}_{4} \mathrm{~F}_{3} \mathrm{NO}: 187.0200$, found: 186.0172. NMR ${ }^{1} \mathrm{H}$ (400 MHz; $\left.\mathrm{CD}_{3} \mathrm{COCD}_{3}, \delta, \mathrm{ppm}\right): 7.0$ (m, 1H, H-7); 7.40 (m, 1H, H-6);7.70 (m, 1H, H-4); 11.20 $(\mathrm{s}, 1 \mathrm{H}, \mathrm{NH}), \mathrm{NMR}^{13} \mathrm{C}\left(100 \mathrm{MHz} ; \mathrm{CD}_{3} \mathrm{COCD}_{3}, \delta, \mathrm{ppm}\right): 110.8(\mathrm{t}, J=247.9 \mathrm{~Hz}, \mathrm{C}-3) ; 112.7$ (C-7); 113.1 (C-7a); 120.2 (td, J = 8.5Hz, C-4); 120.8 (d, J = 23.4 Hz, C-6); 138.7 (td, J = 2.1 Hz, C-3a); 158.2 (d, J = 238.8 Hz, C-5); 165.7 (t, J = 29.0 Hz, C-2), NMR ${ }^{19}$ F (376 MHz; DMSO, $\delta$, ppm): $-111.1\left(\mathrm{CF}_{2}\right) ;-119.0(\mathrm{CF})$.

3.1.6. General Procedure for the Preparation of 3,3-difluoro-1-propylindoline-2-one (14a-e)

Briefly, $2.9 \mathrm{mmol}$ (1 eq) of the corresponding isatins, 13a-e, $4.7 \mathrm{mmol}$ (1.63 eq) of propargyl bromide, $5.5 \mathrm{mmol}(1.9 \mathrm{eq})$ of potassium carbonate, $0.9 \mathrm{mmol}(0.32 \mathrm{eq})$ of sodium iodide and 6 $\mathrm{mL}$ of anhydrous DMF were added in a monotube flask. The progress of the reaction was monitored using TLC (hexane/ethyl acetate 7:3). After $24 \mathrm{~h}$ at room temperature, $5 \mathrm{~mL}$ of $3 \mathrm{~N}$ $\mathrm{HCl}$ solution and ice were added, and a precipitate was formed. This solid was filtered with a vacuum to give the desired product.

The attributes can be summarized as follows:

3,3-difluoro-1-propylindoline-2-one (14a): Yield: 85\%; m.p.: 76-78 ${ }^{\circ} \mathrm{C}$ (lit. 76-78 $\left.{ }^{\circ} \mathrm{C}\right)$ [19]. IR $\left(\mathrm{cm}^{-1}\right)$ : 3250; 2125; 1744; 1304. MS-ESI [M+Na] ${ }^{+}(\%): 230$ (100). MS-GC (70 eV, $\left.m / z, \%\right)$ : 168 (100); 207 (78); 179 (18); 178 (12); 126 (12).

3,3-difluoro-5-methyl-1-propylindoline-2-one (14b): Yield: 94\%; m.p.: 102-105 ${ }^{\circ} \mathrm{C}$ (lit. $\left.102-104{ }^{\circ} \mathrm{C}\right)$ [19]. IR $\left(\mathrm{cm}^{-1}\right): 3247 ; 1748 ; 1298$. MS-GC (70 eV, $\left.m / z, \%\right): 221$ (94); 183 (100); 192 (17); 222 (13); 178 (13).

5-chloro-3,3-difluoro-1-propylindoline-2-one (14c): Yield: 77\%; m.p.: $87-89{ }^{\circ} \mathrm{C}$ (lit. 87-89 $\left.{ }^{\circ} \mathrm{C}\right)$ [17]. IR $\left(\mathrm{cm}^{-1}\right): 3259 ; 2126 ; 1726 ; 1335$. MS-GC (70 eV, $\left.m / z, \%\right): 202$ (100); 241 (92); $243(32) ; 178$ (26).

5-bromo-3,3-difluoro-1-propylindoline-2-one (14d): Yield: 52\%; m.p.: $115-116^{\circ} \mathrm{C}$. IR $\left(\mathrm{cm}^{-1}\right): 3261 ; 1726 ; 1276$. MS-ESI [M+1] ${ }^{+}(\%): 284$ (100). $\mathrm{NMR}^{1} \mathrm{H}\left(400 \mathrm{MHz} ; \mathrm{CD}_{3} \mathrm{COCD}_{3}\right.$, $\delta$, ppm): $3.42\left(\mathrm{t}, J=8.8 \mathrm{~Hz}, 1 \mathrm{H}, \mathrm{H}-3^{\prime}\right) ; 4.60\left(\mathrm{~d}, J=2.5 \mathrm{~Hz}, 2 \mathrm{H}, \mathrm{H}-1^{\prime}\right) ; 7.30(\mathrm{~d}, J=8.5 \mathrm{~Hz}, 1 \mathrm{H}$, 
$\mathrm{H}-7) ; 7.90(\mathrm{dt}, J=1.1 \mathrm{~Hz}, \mathrm{H}-6) ; 8.0(\mathrm{~d}, J=1.8 \mathrm{~Hz}, 1 \mathrm{H}, \mathrm{H}-4) . \mathrm{NMR}^{13} \mathrm{C}\left(100 \mathrm{MHz} \mathrm{CD}_{3} \mathrm{COCD}_{3}\right.$, $\delta, \mathrm{ppm}): 29.6\left(\mathrm{C}-1^{\prime}\right) ; 75.6\left(\mathrm{C}-3^{\prime}\right) ; 76.5\left(\mathrm{C}-2^{\prime}\right) ; 110.2(\mathrm{t}, J=248.6 \mathrm{~Hz}, \mathrm{C}-3) ; 113.4(\mathrm{C}-7) ; 116.0$ (C-5); 120.6 (t, J = 22.9 Hz, C-3a); 127.8 (C-4); 137.0 (C-6); $141.4(\mathrm{t}, J=6.8 \mathrm{~Hz}, \mathrm{C}-7 \mathrm{a}) ; 163.1(\mathrm{t}$, $J=29.9 \mathrm{~Hz}, \mathrm{C}-2) . \mathrm{NMR}^{19} \mathrm{~F}(376 \mathrm{MHz}$; DMSO, $\delta, \mathrm{ppm}):-110.6$.

5-fluoro-3,3-difluoro-1-propylindoline-2-one (14e): Yield: 62\%. IR ( $\left.\mathrm{cm}^{-1}\right)$ : 3305; 1753; 1293; 1087. MS-ESI [M+1] ${ }^{+}$(\%): 226 (100). MS-GC (70 eV, $\left.m / z, \%\right): 186$ (100); 225 (78); 158 (16); 144 (15); 196 (12). NMR ${ }^{1} \mathrm{H}$ (400 MHz; $\left.\mathrm{CD}_{3} \mathrm{COCD}_{3}, \delta, \mathrm{ppm}\right): 3.41$ (m, 1H, H-3'); $4.61\left(\mathrm{~d}, J=2.5 \mathrm{~Hz}, 2 \mathrm{H}, \mathrm{H}-1^{\prime}\right) ; 7.37(\mathrm{~m}, 1 \mathrm{H}, \mathrm{H}-7) ; 7.57(\mathrm{~m}, 1 \mathrm{H}, \mathrm{H}-6) ; 7.8(\mathrm{~d}, J=1.8 \mathrm{~Hz}, 1 \mathrm{H}$, H-4). NMR ${ }^{13} \mathrm{C}\left(100 \mathrm{MHz} ; \mathrm{CD}_{3} \mathrm{COCD}_{3}, \delta, \mathrm{ppm}\right): 29.5\left(\mathrm{C}-1^{\prime}\right) ; 75.4\left(\mathrm{C}-3^{\prime}\right) ; 76.5\left(\mathrm{C}-2^{\prime}\right) ; 112.8(\mathrm{t}$, $J=243.9 \mathrm{~Hz}, \mathrm{C}-3) ; 113.1(\mathrm{C}-7) ; 119.8(\mathrm{td}, J=8.7 \mathrm{~Hz}, \mathrm{C}-4) ; 120.8(\mathrm{~d}, J=23.7 \mathrm{~Hz}, \mathrm{C}-3 \mathrm{a}) ; 138.2$ (td, J = 2.0 Hz, C-6); 157.6 (t, $J=2.5 \mathrm{~Hz}, \mathrm{C}-5) ; 160.0$ (t, J = 2.6 Hz, C-7a); 163.4 (t, J = 29.7 Hz, C-2). NMR ${ }^{19} \mathrm{~F}$ (376 MHz; DMSO, $\left.\delta, \mathrm{ppm}\right):-110.6$.

\subsection{Biological Evaluation}

\subsubsection{Cell Lines}

The A549 cell line (CCL-185 ${ }^{\mathrm{TM}}$; ATCC, Manassas, VA, USA), from human lung carcinoma epithelium, and the K562 cell line (Rio de Janeiro Cell Bank, BCRJ: 0126, Rio de Janeiro, RJ, Brazil), a human chronic myeloid cell line expressing the BCR-ABL1 protein, were grown in RPMI-1640 culture medium (Merck, Darmstadt, Hesse, Germany) supplemented with 10\% heat-inactivated fetal bovine serum (FBS; Vitrocell, Campinas, SP, Brazil). WSS-1 [WS-1] (ATCC CRL-2029 ${ }^{\mathrm{TM}}$ ) human kidney epithelial cells were grown in high-glucose DMEM (Vitrocell) supplemented with 10\% FBS (Vitrocell). All cell lines were maintained at $37{ }^{\circ} \mathrm{C}$ under $5 \% \mathrm{CO}_{2}$, in a water jacket $\mathrm{CO}_{2}$ incubator (Forma Series II incubator, Thermo Fisher Scientific, Waltham, MA, USA).

\subsubsection{Cytotoxicity Assays}

The cytotoxic activity of imatinib and its analogs was evaluated by a fluorimetric assay, based on the metabolic reduction of resazurin to resorufin. Briefly, cells were seeded in 96-well microplates $\left(2 \times 10^{4}\right.$ cells /well for K562 cells and $5 \times 10^{4}$ cells /well for A549 and WSS-1 cells) and incubated with $10 \mu \mathrm{M}$ of the test compounds for $48 \mathrm{~h}$ at $37^{\circ} \mathrm{C}$. A resazurin (Merck, Darmstadt, Hesse, Germany) solution (final concentration: $0.01 \mathrm{mg} / \mathrm{mL}$ ) was added to each well before the end of the experiment, at $1 \mathrm{~h}$ before concluding for K562 cells, and $2 \mathrm{~h}$ before concluding for A549 and WSS-1 cells. Fluorescence readings $\left(\lambda_{\mathrm{ex}}=560 \mathrm{~nm}, \lambda_{\mathrm{ex}}=590 \mathrm{~nm}\right)$ were carried out immediately after the addition of resazurin $\left(t_{0}\right)$ and at the end of the experiment $\left(t_{n}\right)$ with a FlexStation 3 Benchtop multimode microplate reader (Molecular Devices, San Jose, CA, USA). Resorufin fluorescence was calculated by subtracting the fluorescence measured at $t_{0}$ from that at $t_{n}$. Cell viability was expressed as percentages, considering the mean resorufin fluorescence of the control wells (cells incubated with only DMSO) to be $100 \%$. Tests were performed in triplicate. The same assay was carried out to generate 8-10 points concentration-response curves (300 $\mu \mathrm{M}$ top compound concentration). From this analysis it was possible to calculate the $\mathrm{IC}_{50}$ (concentration of a compound the reduces in $50 \%$ cell viability) values.

\subsubsection{Kinase Inhibition Assays}

The ABL1 inhibitory activity assay was performed using the ABL1 Kinase Enzyme System and ADP-Glo ${ }^{\mathrm{TM}}$ Kinase assay kit (Promega, Madison, WI, USA) according to the manufacturer's instructions. The general procedure was as follows: kinase (2.5 ng/reaction) was incubated with the substrate $(1 \mu \mathrm{g})$, compound $(0.5$ and $10 \mu \mathrm{M})$, and ATP $(25 \mu \mathrm{M})$ in a commercial buffer solution with a reaction volume of $5 \mu \mathrm{L}$. In every experiment, noenzyme and no-compound control reactions were included to represent the background luminescence ( $0 \%$ activity) and uninhibited kinase activity (100\% activity), respectively. The assay 384-well plate was incubated at room temperature for $1 \mathrm{~h}$. Afterward, $5 \mu \mathrm{L}$ of ADP-Glo reagent was added to each well to stop the reaction and consume the remaining ADP within $40 \mathrm{~min}$. At the end of this period, $10 \mu \mathrm{L}$ of kinase detection reagent was added 
to each well and incubated for 30 min to produce a luminescence signal. Kinase activity assays were performed in triplicate at each inhibitor concentration. The luminescence was measured in a FlexStation 3 (Molecular Devices, San Jose, CA, USA) plate reader. The percentage of kinase activity (KA) was calculated by subtracting the average no-enzyme control luminescence values from all kinase-containing reactions with or without the compound, then converting these net luminescence values into percentage activity, based on the no-compound control reactions representing 100\% kinase activity. Inhibition (I, \%) was calculated as $100 \%-K A$. Imatinib at $10 \mu \mathrm{M}$ was used as a positive inhibition control.

\subsubsection{Statistical Analysis}

$\mathrm{IC}_{50}$ values were calculated by a four-parameter logistic curve function, using GraphPad Prism version 6.01 for Windows (GraphPad Software, San Diego, CA, USA, www. graphpad.com) (accessed on 29 December 2021).

\section{Conclusions}

Many patients have presented with resistance to TKIs, which has led to the search for new therapeutic options. In this work, ten new imatinib analogs were synthesized, and their antiproliferative activities against cancer (K562 and A549) and normal (WSS-1) human cell lines were evaluated. A molecular hybridization strategy was used to obtain compounds 2a-e and 3a-e with PAPP and isatin scaffold units, spaced with a $1 H$-1,2,3-triazole ring and with different substituents at the C-5 carbon of isatin. Biological evaluations showed that compound $\mathbf{3 b}$ exhibited the highest cytotoxic activity against the K562 cell line, with an $\mathrm{IC}_{50}$ value of $35.8 \mu \mathrm{M}$. Evaluations of the A549 cell line showed that $\mathbf{3 a}, \mathbf{3 c}$ and $\mathbf{3 d}$ were more potent and selective than imatinib. These results indicate the importance of the $\mathrm{CF}_{2}$ group since, in compounds $2 \mathrm{a}-\mathbf{e}$, the absence of this group led to a loss of activity. These data suggest that these compounds can be used as prototypes for other studies of compounds against CML and lung cancer. The imatinib analogs (2a-e and $\mathbf{3 a - e})$ were also investigated for the inhibition of ABL1 enzyme activity and did not show inhibitory effects at all concentrations. In the future, other enzymatic studies will be carried out, including work with the BCR-ABL1 enzyme.

Supplementary Materials: The following are available, Figure S1: ESI-MS of compound 10; Figure S2-S6: GC-MS of compound 12a-e; Figure S7-S22: HRMS, ${ }^{1} \mathrm{H}$ NMR, ${ }^{13} \mathrm{C}$ NMR of compound 2a-e; Figure S23-S32: HRMS, ${ }^{1} \mathrm{H}$ NMR, ${ }^{13} \mathrm{C}$ NMR of compound 13a-e; Figure S33-S41: HRMS, ${ }^{1} \mathrm{H}$ NMR, ${ }^{13} \mathrm{C}$ NMR of compound 14a-e; Figure S42-S61: HRMS, ${ }^{1} \mathrm{H}$ NMR, ${ }^{13} \mathrm{C}$ NMR of compound 3a-e.

Author Contributions: Experimental work, A.O. and S.M.; bibliographic research, L.P.; supervision in organic synthesis, M.B.; supervision of in vitro biological evaluation, J.N., R.D. and F.S.-J.; project management and financing acquisition, N.B. All authors have read and agreed to the published version of the manuscript.

Funding: This study was financed in part by the Coordenação de Aperfeiçoamento de Pessoal de Nível Superior-Brasil (CAPES)-Finance Code 001, by grants awarded to N.B. (E-26/202.805/2017) from the Foundations for Research of the State of Rio de Janeiro (FAPERJ) ("Cientista do Nosso Estado"), and fellowship grants from the National Council of R\&D of Brazil (CNPq) awarded to N.B. (306193/2018-3) and F.P.S.J. (304059/2018-8).

Informed Consent Statement: Not applicable.

Data Availability Statement: Not applicable.

Acknowledgments: The authors wish to thank Cristalia SA for the PAPP (9) donation. This study was financed in part by the Coordenação de Aperfeiçoamento de Pessoal de Nível Superior-Brasil (CAPES)-Finance Code 001 (A.O.). The authors would like to thank the National Council of R\&D of Brazil (CNPq) for fellowship grants to N.B. (306193/2018-3) and F.P.S.J. (304059/2018-8). They also thank the Foundations for Research of the State of Rio de Janeiro (FAPERJ) for the fellowships granted ("Cientista do Nosso Estado") to N.B. (E-26/202.805/2017) and scholarship for A.O.

Conflicts of Interest: The authors declare no conflict of interest. 
Sample Availability: Samples of the compounds are available from the authors.

\section{References}

1. Vollenhoven, R.F.V. Kinase inhibition: A new therapeutic principle in rheumatology. Rheumatology 2015, 1, 511-517. [CrossRef]

2. Jiao, Q.; Bi, L.; Ren, Y.; Song, S.; Wang, Q.; Wang, Y. Advances in studies of tyrosine kinase inhibitors and their acquired resistance. Mol. Cancer 2018, 171, 36. [CrossRef] [PubMed]

3. Ferguson, F.M.; Gray, N.S. Kinase inhibitors: The road ahead. Nat. Rev. Drug Discov. 2018, 17, 353-377. [CrossRef] [PubMed]

4. Knight, T.G.; Grunwald, M.R.; Copelan, E.A. Chronic myeloid leukemia (CML). In Concise Guide to Hematology; Springer International Publishing: Cham, Switzerland, 2018; pp. 313-322.

5. Westerweel, P.E.; Te Boekhorst, P.A.W.; Levin, M.D.; Cornelissen, J.J. Novas abordagens e combinações de tratamento para o tratamento da leucemia mielóide crônica. Front Oncol. 2019, 9, 665. [CrossRef] [PubMed]

6. Azevedo, L.D.; Bastos, M.M.; Vasconcelos, F.C.; Hoelz, L.V.B.; Junior, F.P.S.; Dantas, R.F.; De Almeida, A.C.M.; Oliveira, A.P.; Gomes, L.C.; Maia, R.C.; et al. Imatinib derivatives as inhibitors of K562 cells in chronic myeloid leukemia. Med. Chem. Res. 2017, 26, 2929-2941. [CrossRef]

7. Rossari, F.; Minutolo, F.; Orciuolo, E. Past, present, and future of Bcr-Abl inhibitors: From chemical development to clinical efficacy. J. Hematol. Oncol. 2018, 11, 84. [CrossRef] [PubMed]

8. Luciano, L.; Annunziata, M.; Attolico, I.; Di Raimondo, F.; Maggi, A.; Malato, A.; Martino, B.; Palmieri, F.; Pane, F.; Sgherza, N.; et al. The multi-tyrosine kinase inhibitor ponatinib for chronic myeloid leukemia: Real-world data. Eur. J. Haematol. 2020, $105,3-15$. [CrossRef] [PubMed]

9. Wee, P.; Wang, Z. Epidermal Growth Factor Receptor Cell Proliferation Signaling Pathways. Cancers 2017, 9, 52. [CrossRef] [PubMed]

10. Gaber, A.A.; Bayoumi, A.H.; El-morsy, A.M.; Sherbiny, F.F.; Mehany, A.B.M.; Eissa, H.I. Design, synthesis and anticancer evaluation of ${ }^{1} \mathrm{H}$-pyrazolo[3,4- $d$ ]pyrimidine derivatives as potent EGFR ${ }^{\mathrm{WT}}$ and EGFR ${ }^{\mathrm{T} 790 \mathrm{M}}$ inhibitors and apoptosis inducers. Bioorg. Chem. 2018, 80, 375-395. [CrossRef] [PubMed]

11. Fabbro, D.; Jacob, S.W.C.; Moebitz, H. Ten things you should know about protein kinases: IUPHAR Review 14. Br. J. Pharmacol. 2015, 172, 2675-2700. [CrossRef] [PubMed]

12. Pimentel, L.C.F.; Hoelz, L.V.B.; Canzian, H.F.; Branco, F.S.C.; Oliveira, A.P.; Campos, V.R.; Junior, F.P.S.; Dantas, R.F.; Resende, J.A.L.C.; Cunha, A.C.; et al. Phenylamino pyrimidine-1,2,3-triazole derivatives as analogs of imatinib: Searching for novel compounds against chronic myeloid leucemia. Beilstein J. Org. Chem. 2021, 17, 2260-2269. [CrossRef] [PubMed]

13. Pimentel, L.C.F.; Cunha, A.C.; Hoelz, L.V.B.; Canzian, H.F.; Marinho, D.I.L.F.; Boechat, N.; Bastos, M.M. Phenylamino-pyrimidine (PAP) Privileged Structure: Synthesis and Medicinal Applications. Curr. Topics Med. Chem. 2020, 20, 227-243. [CrossRef] [PubMed]

14. Türe, A.; Ergül, M.; Ergül, M.; Altun, A.; Küçükgüzel, İ. Design, synthesis, and anticancer activity of novel 4-thiazolidinonephenylaminopyrimidine hybrids. Mol. Divers. 2020, 25, 1025-1050. [CrossRef] [PubMed]

15. Varun, C.; Sonam, N.; Kakkar, R. Isatin and its derivatives: A survey of recent syntheses, reactions, and applications. Med. Chem. Comum. 2019, 10, 351-368. [CrossRef] [PubMed]

16. Zhang, Q.; Teng, Y.; Yuan, Y.; Ruan, T.; Wang, Q.; Gao, X.; Zhou, Y.; Han, K.; Yu, P.; Lu, K. Synthesis and cytotoxic studies of novel 5-phenylisatin derivatives and their anti-migration and anti-angiogenic evaluation. Eur. J. Med. Chem. 2018, 156, 800-814. [CrossRef] [PubMed]

17. Li, Y.T.; Wang, J.H.; Pan, C.W.; Meng, F.F.; Chu, X.Q.; Ding, Y.; Qua, W.Z.; Li, H.; Yang, C.; Zhang, Q.; et al. Syntheses and biological evaluation of 1,2,3-triazole and1,3,4-oxadiazole derivatives of imatinib. Bioorg. Med. Chem. Lett. 2016, 26, 1419-1427. [CrossRef] [PubMed]

18. Shijie, C.; Long, H.; Xuewei, W.; Xianfeng, G.; Hua, Z. Synthesis and Cytotoxic Activity of Imatinib Derivatives. Chin. J. Org. Chem. 2015, 35, 2377. [CrossRef]

19. Boechat, N.; Bastos, M.M.; Souza, T.M.L.; Leite, D.I.; Bernardino, A.M.R. Isatin-Derived Compounds, Use of the Compounds for the Treatment of AIDS and Method of Treatment Using These Compounds. BR Patent BR2016000050, 10 May 2016.

20. Fawzy, I.M.; Youssef, K.M.; Lasheen, D.S.; Ismail, N.S.M.; Abouzid, K.A.M. Design, synthesis and 3D QSAR based pharmacophore study of novel imatinib analogs as antitumor-apoptotic agents. Future Med. Chem. 2018, 10, 1421-1433. [CrossRef] [PubMed]

21. Boechat, N. Isatinas: Mais uma prova de versatilidade. Fluorodenitração: Uma alternativa. Tese de Doutorado em Química Orgânica, Universidade Federal do Rio de Janeiro, Rio de Janeiro, Brazil, 1996.

22. Torres, J.C.; Garden, S.J.; Pinto, A.C.; Da Silva, F.S.Q.; Boechat, N. A synthesis of 3-fluoroindoles and 3,3-difluoroindolines by reduction of 3,3-difluoro-2-oxindoles using a borane tetrahydrofuran complex. Tetrahedron 1999, 55, 1881-1892. [CrossRef] 